\title{
Energy-efficient resource allocation for ultra-dense licensed and unlicensed dual-access small cell networks
}

\author{
Adnan Shahid, Senior Member, IEEE, Vasilis Maglogiannis, Student Member, IEEE, \\ Irfan Ahmed, Senior Member, IEEE, Kwang Soon Kim, Senior Member, IEEE, Eli De Poorter, \\ Ingrid Moerman
}

\begin{abstract}
In this study, an energy-efficient self-organized framework for sub-channel allocation and power allocation is presented for ultra-dense small cell networks, which can operate in both licensed and unlicensed bands. In order to protect legacy WiFi devices (operating in unlicensed bands), we consider the LTE operation in unlicensed bands based on CSAT, in which 'ON' and 'OFF' duty cycle approach is utilized. On the other hand, there are severe interference management problems among small cells (operating in licensed and unlicensed bands) and between macro cells and small cells (operating in licensed bands) due to co-channel and ultra-dense deployment of small cells. This article proposes a self-organized optimization framework for the allocation of sub-channels and power levels by exploiting a non-cooperative game with the objective to maximize the energy efficiency of dual-access small cells without creating harmful impact on coexisting network entities including macro cell users, small cell users, and legacy WiFi devices. Simulation results show that the proposed scheme outperforms $(6 \%$ and $11 \%)$ and $(8 \%$ and $18 \%)$ the round-robin and the spectrum-efficient schemes, respectively for two different small cell scenarios. In addition, it is shown that for less CSI estimation errors $\varsigma=0.02$, the maximum performance degradation of the proposed scheme is reasonably small (5.5\%) as compared to the perfect CSI.
\end{abstract}

Index Terms-Self-organization, sub-channel allocation, power allocation, non-cooperative game, small cells, licensed band, unlicensed band.

\section{INTRODUCTION}

$\mathrm{D}$ UE to the proliferation of real-time bandwidth hungry applications such as video streaming, online gaming and mobile computing, there is a sharp increase in the bandwidth demand. According to a recent report by Cisco Global Mobile Data Traffic Forecast [1], there will be around 11 billion mobile devices by 2019 which is above the world's expected population at that time (7.6 billion). Also, it is reported by the industry that cellular traffic is doubling every year; therefore, cellular network should be able to deal with the 1000x increase in cellular traffic by 2020 [2]. This increase in the traffic demand imposes critical challenges in terms of meeting the quality of service (QoS) requirements from the existing cellular network architecture. The limiting factor here is the licensed spectrum, which is a real bottleneck in meeting the increasing traffic demand. On the other hand, the continuous increase in the cellular traffic is also a serious threat to the energy consumption of future cellular networks and it has a direct impact on the operating cost (OPEX) of cellular operators.

- A. Shahid (corresponding author), V. Maglogiannis, E. De Poorter and I. Moerman are with the IDLab, Department of Information Technology, Ghent University - imec, iGent Tower, Technologiepark - Zwijnaarde 15, B-9052 Ghent, Belgium. Email: adnan.shahid@ugent.be

- I. Ahmed is with the Department of Electrical Engineering, Higher Colleges of Technology, Ruwais Campus, Abu Dhabi, United Arab Emirates.

- K.S. Kim is with the Department of Electronics and Electrical Engineering, Yonsei University, 50 Yonsei-ro, Seodaemun-gu, Seoul 120-749, Republic of Korea.

Manuscript received Oct 08, 2019; revised -.
Thereby, an energy-efficient design for the future cellular networks is needed.

To cope with the ever increasing traffic demands, various innovative technologies / methodologies have been developed such as non-orthogonal multiple access (NOMA), multiple-input multiple-output (MIMO), carrier aggregation (CA), small cells, context awareness, etc [3]-[6]. Among various options, small cells, which are deployed as underlay within macro cells, boost the coverage and capacity by reusing the licensed spectrum. Qualcomm states that the dense deployment of small cells is the foundation of the $1000 x$ vision of the fifth generation $(5 \mathrm{G})$ cellular network [7]. This ultra-dense deployment of small cells increases the coverage per square kilometer and the capacity by reusing the spectrum. More specifically, this ultradense deployment contributes a 56-fold in the 1000-fold traffic demand [8]. Although this ultra-dense deployment holds great promise for enhancing the spectrum efficiency by exploiting both spatial and universal frequency reuse, the co-location of small cells underlaid in macro cells creates severe interference management problems. In this context, recent scientific literature focuses on the interference management for enhancing the spectrum efficiency [9]-[11], while energy efficiency is almost neglected. The increase in the spectrum efficiency corresponds to high throughput but at the cost of large energy consumption which is mostly not suitable for energy-aware networks or constrained devices [12]. Recently, energy efficiency, which is defined as information bits per unit of transmit energy 
(bits/J), has received significant attention from industry, 111 academia, and standardization bodies. Some studies have 112 revealed that energy efficiency will be an integral part of ${ }_{113}$ the 5G cellular networks, especially for the ultra-dense 114 small cell environment for meeting the QoS requirements 115 [8]. Thus, we can say that both spectrum efficiency and 116 energy efficiency will be critical for interference manage- 117 ment in the ultra-dense small cell environment, which is 118 confirmed when looking at the $5 \mathrm{G}$ vision and requirements 119 [13]. Hu et al. [13] propose a cooperative mechanism for 120 finding a balance between energy efficiency and spectrum 121 efficiency, but it is not suitable for the ultra-dense en- 122 vironment because it incurs huge information exchange 123 overhead. On the contrary, this work aims to propose an 124 efficient and a less complex scheme which does not require 125 cooperation and thus, minimizes the information exchange 126 overhead.

Also, WiFi hotspot has been successfully utilized for ${ }_{128}$ offloading the cellular traffic from licensed band to unli- ${ }_{129}$ censed band [14]. Considering the benefits of small cells ${ }_{130}$ and WiFi hotspots, one natural enhancement of small cells ${ }_{131}$ is to operate in both licensed (e.g. LTE or third generation ${ }_{132}$ $(3 \mathrm{G})$ ) and unlicensed (e.g. WiFi) bands simultaneously. We ${ }_{133}$ refer to these enhanced small cells as the dual-access small ${ }_{134}$ cells since they can operate in both licensed and unlicensed ${ }_{135}$ bands. The coexistence of LTE and WiFi in unlicensed ${ }_{136}$ bands has recently gained significant attention and in ${ }_{137}$ this regard, two prominent standardization efforts have ${ }_{138}$ been made: LTE in LTE in unlicensed spectrum (LTE-U) 139 [15] and Licensed Assisted Access (LAA) [16]. LTE-U was 140 standardized by the LTE-U forum which is based on ${ }_{141}$ Carrier Sense Adaptive Transmission (CSAT) for selecting ${ }_{142}$ 'ON' and 'OFF' duty cycles according to WiFi transmis- ${ }_{143}$ sion occupancy. Whereas, LAA was standardized by the ${ }_{144}$ 3rd Generation Partnership Project (3GPP) in Release 13145 and uses the listen-before-talk (LBT) technique (similar to ${ }_{146}$ WiFi). In this work, we consider LTE-U based CSAT and ${ }_{147}$ leave LAA for future investigations. The term dual access 148 small cell that we use in this work can be termed as LTE-U ${ }_{149}$ based on CSAT. Later in this article, we will use the terms 150 dual access small cells and LTE-U interchangeably.

The rapid evolution of LTE-U raises critical concerns on 152 the coexistence of LTE and WiFi. Primarily, the coexistence ${ }_{153}$ of LTE and WiFi is a challenging problem. The main chal- ${ }_{154}$ lenge is to find a balance between the operation of LTE and 155 the proprietary technologies including WiFi that operate 156 in unlicensed bands. Traditionally, LTE was designed to 157 operate centrally as a non-contention technology and has ${ }_{158}$ no concern with cross-tier interference i.e., interference 159 from other technologies. In addition, LTE is a streaming 160 technology which means that even in the absence of ${ }_{161}$ data traffic, control and reference signals are transmitted ${ }_{162}$ over-the-air. On the contrary, WiFi is a contention-based ${ }_{163}$ technology and it only transmits when the channel is not ${ }_{164}$ occupied. This makes WiFi an ideal candidate to operate 165 in unlicensed bands. Due to these fundamental differences, 166 the operation of traditional LTE in unlicensed bands could ${ }_{167}$ have a significant impact on the performance of WiFi [17]. 168 Even though due to the adoption of CSAT in LTE, its 169 operation can degrade the WiFi performance [18]. We also 170 observed the similar performance degradation trend by 171 experimentally validating the LTE impact on WiFi by using open source LTE implementation [19]. This degradation mainly because of two factors: (i) the incompatible LTE-U duty cycle mechanism with WiFi devices and (ii) the lack of an efficient coexistence mechanism when LTE and WiFi devices are far apart from each other and the received power is low [20]. To address such coexistence problems, this paper considers dual-access small cells based on the CSAT approach which provides a coexistence mechanism by using 'ON' and 'OFF' duty cycles for LTE and other unlicensed technologies including WiFi [21]. In the LTE-U 'ON' period, each dual-access small cell base station (SBS) utilizes sub-channels from both licensed and unlicensed bands while the legacy WiFi devices backoff due to the adoption of the carrier-sense multiple access with collision avoidance (CSMA/CA) protocol. While in the 'OFF' period, the SBSs do not transmit and provide transmission opportunities to the legacy WiFi devices. In this work, we assume that 'ON' and 'OFF' periods are determined by each dual-access SBS in a self-organizing way and are based on the transmission occupancy of the legacy WiFi devices. However, this creates another challenge of interference management specially in the ' $\mathrm{ON}^{\prime}$ period due to the co-channel operation of (i) small cells and macro cells in licensed bands and (ii) small cells and the legacy WiFi devices in unlicensed bands. In such a coexisting environment, meeting the QoS requirements is a critical challenge and for this, we aim to solve this by investigating sub-channel allocation and power allocation for dual-access small cell networks. Generally, a centralized architecture can be an option here for managing the interference optimally. However, such a centralized control is not applicable in the considered dense environment due to its lack of scalability and increased information exchange overhead.

Motivated from the above-mentioned concerns, in this work, a self-organized sub-channel allocation and power allocation scheme for the downlink of dual-access small cell networks is modeled as a non-cooperative game. Since for an ultra-dense environment centralized management approaches are not appropriate, we aim to propose a self-organizing solution and for this game-theoretic approaches are most suited. Such a game-theoretic approach provides a mathematical tool for modeling an optimization problem [22], [23]. The main goal of the proposed scheme is to enhance the energy efficiency of small cell networks while meeting the throughput requirements of small cell users, macro cell users, and the legacy WiFi devices. Thus, energy efficiency is considered as the QoS criterion of the proposed scheme. In the proposed noncooperative game, the dual-access SBSs are the players of the game while the sub-channels and the power levels are the strategies of the game. Since the goal is to design an energy-efficient self-organized resource management scheme, the problem is formulated as follows: each player of the game (SBS) during the 'ON' period selects the subchannels (from licensed and unlicensed bands) and the power levels for enhancing its energy-efficiency without creating harmful impact on the coexisting networking entities including small cell users, macro cell users, and the legacy WiFi devices. In addition, we propose that ' $\mathrm{ON}^{\prime}$ 
and 'OFF' periods are selected by each dual-access SBS in 230 a self-organizing way based on the legacy WiFi devices 231 occupancy. Concerning the complexity of the joint subchannel allocation and power allocation problem, the joint problem is decomposed into two sub-problems: the subchannel allocation and the power allocation. In the subchannel allocation step, the allocation of sub-channels from both licensed and unlicensed bands is carried out with the concern of minimizing the impact of cross-tier and co-tier interference components. Where the cross-tier interference is the interference among different tiers i.e., small cells, macro cell, and legacy WiFi devices while the co-tier is between the same tier. In the power allocation step, the selection of power levels on the sub-channels (selected in the previous step) is carried out by exploiting dualdecomposition Lagrangian multipliers.

\subsection{Contributions}

The main contributions of our work are summarized as under:

1) A framework is proposed for solving the sub-channel allocation and power allocation problem in the 250 downlink of dual-access small cells which operate in 251 both licensed and unlicensed bands. The framework 252 aims to maximize the energy efficiency of small cells 253 using a non-cooperative game without compromising 254 the QoS requirements of small cell users, macro cell 255 users, and the legacy WiFi devices.

2) Since the energy-efficient sub-channel allocation 258 and power allocation is in a fractional form and a 259 non-convex optimization problem, traditional convex 260 optimization techniques cannot be applied for finding 261 the optimal solution. We first transform the problem ${ }^{262}$ into its equivalent subtractive form using the properties 263 of non-linear fractional integer programming and then, 264 propose an efficient and a less complex iterative 265 algorithm for solving the sub-channel allocation and 266 the power allocation individually. In addition, the ${ }^{267}$ proposed iterative algorithm is shown to converge 268 to the optimal energy efficiency and to the Nash 269 Equilibrium point.

3) Simulations results are carried out in terms of energy 272 efficiency and spectrum efficiency to compare the pro- 273 posed scheme utilizing both licensed and unlicensed 274 bands with the comparative counterparts: a) the pro- 275 posed scheme utilizing only licensed bands, b) the 276 round robin non-cooperative power optimization game 277 (RR-NPOG) [24], and c) the spectrum efficient scheme. 278 The performance improvement of the proposed scheme 279 as compared to the counterparts $(\mathrm{a}, \mathrm{b}$, and $\mathrm{c})$ increases 280 from $5 \%, 7.6 \%$, and $10.9 \%$ to $7.73 \%, 11.04 \%$, and $17.06 \%$, 281 respectively, with the increase of the small cell density 282 from 20 to 40. In addition, the proposed scheme is eval- 283 uated for different levels of imperfection in estimating 284 CSI: perfect CSI and imperfect CSI. Which concludes 285 that for less CSI estimation errors $\varsigma=0.02$, the maxi- ${ }^{286}$ mum performance degradation of the proposed scheme ${ }_{287}$ is reasonable small $(5.5 \%)$ as compared to the perfect CSI.

\section{Related Work}

The concept of aggregating radio resources is nowadays an active research topic and in this context, the literature includes several works that address the coexistence of LTE and WiFi. On the other hand, an energy-efficient design specially for a dense environment has recently gained significant attention from the scientific community. Based on this, we present the related work in three parts: i) energy-efficient resource allocation in wireless networks, ii) general approaches for LTE and WiFi coexistence, and iii) game-theoretic approaches for LTE and WiFi coexistence.

\subsection{Energy-efficient resource allocation in wireless networks}

Li et al. [12] present a comprehensive survey on an energyefficient design of wireless networks and highlight key areas such as information-theoretic analysis, MIMO techniques, relaying, and resource allocation for meeting the QoS requirements. Due to the explosion of high data rate applications in future wireless networks, more energy will be consumed for meeting such requirements. Therefore, it is imperative to design energy-efficient mechanisms while satisfying the requirements. On the other hand, future wireless networks will be extremely dense and in this regard, centralized management architectures are not suitable due to their lack of scalability and increased information exchange overhead. Meshkati et al. [25] present a detailed overview of energy-efficient resource allocation mechanisms in wireless networks using game theoretic approaches. In a dense environment, game-theoretic approaches are most suited due to their ease of scalability and reduced information exchange overhead. Xie et al. [26] investigate and formulate an energy-efficient resource allocation problem for heterogeneous femto cell cognitive radio networks cells using a Stackelberg game. It only considers co-tier interference and for cross-tier, it is assumed that a spectrum resource borrowed from a primary network can only be allocated to a single femto base station. This assumption is not valid for a dense environment. Arani et al. [27] present a self-organized mechanism for joint channel and power allocation with the concern of maximizing the energy efficiency of heterogeneous networks using a non-cooperative game. It only considers co-channel interference but in a dense environment where LTE and WiFi operate in similar unlicensed bands, crosschannel interference also becomes important. Considering the importance of cross-channel interference, we include it in the problem formulation and by doing so better spectrum decisions can be made. Samarakoon et al. [28] propose a joint power control and user scheduling scheme for enhancing the energy efficiency of ultra-dense small cell networks using a dynamic stochastic game. It does not address the coexistence problem of LTE and $\mathrm{WiFi}$, which is the prime focus of our work. Also, the scheme in [28], cannot be directly applied to solve the formulated problem in this work. 


\subsection{General approaches for LTE and WiFi coexistence ${ }^{347}$}

Zakrzewska et al. [29] propose an idea of splitting the control plane (C-plane) and the user data plane (U-plane) where the C-plane of small cells is provided by a macro base station (MBS) while the U-plane is provided by SBSs. This splitting concept can be employed in dual-access small cells which can operate in both licensed and unlicensed bands. However, the authors do not consider interference management, which is indeed of vital importance when considering coexisting and dense environments. Khawer et al. [30] propose an interference coordination scheme for a CSAT gating mechanism in LTE-U and illustrate multioperator network performance. Lui et al. [31] propose a resource allocation scheme for balancing the traffic between licensed and unlicensed bands while the WiFi throughput is maintained by an optimization framework. He et al. [32] present a novel proportional fair scheme for a fair coexistence of LTE and WiFi is proposed. Despite of the interesting results [29]-[32], all the focus is on spectrum efficiency by managing or avoiding the interference while energy efficiency is ignored and the solutions in these propositions cannot be directly converted to energy efficiency problems. In addition, all the works focus on a centralized control environment which is not suitable in a ultra-dense environment as it poses formidable computational complexity.

\subsection{Game-theoretic approaches for LTE and WiFi co- existance}

Etkin et al. [33] propose a sharing between LTE and ${ }_{377}$ WiFi using a repeated non-cooperative game. A similar 378 scheme with an extended model is proposed in [34] using 379 a repeated non-cooperative game. The authors in [35], 380 [36] propose a spectrum allocation problem in unlicensed 381 bands using a non-cooperative game in which the base ${ }_{382}$ stations are the players and the spectrum resources are the ${ }_{383}$ strategies of the game. The goal in their proposition is to 384 optimize the spectrum efficiency for both downlink and 385 uplink. Thakur et al. in [37] present a resource allocation 386 and a cell selection mechanism for femto cell networks op- 387 erating in licensed and unlicensed bands. For the resource 388 allocation scheme, they propose a non-cooperative game 389 and a Q-learning framework for LTE operating in licensed and unlicensed bands. Finally, they combine the resource allocation scheme with the cell selection and show the performance improvements in terms of throughput and energy efficiency. However, they do not consider co-tier 392 and cross-tier interference which is a challenging problem 393 in a dense environment. In addition, they model the re- 394 source allocation problem while maximizing the through- 395 put instead of energy efficiency. Despite some efforts on 396 the exploitation of game-theoretic approaches for the LTE 397 and WiFi coexistence, all the propositions consider only 398 the spectrum efficiency as the performance objective and 399 the ultra-dense environment is not considered. In addition, 400 the propositions cannot be directly converted to energy 401 efficiency problems in order to achieve the required per- ${ }^{402}$ formance.

According to the best of our knowledge, a self- 404 organized framework for an energy-efficient sub-channel 405 allocation and power allocation for ultra-dense small cell networks which can satisfy the minimum QoS requirements of small cell users, macro cell users, and legacy WiFi devices, has not been investigated so far. In addition, the extension of the results [26], [33]-[37] cannot lead to a reduced complexity iterative sub-channel and power allocation solution. We proposed a similar energyefficient self-organizing framework for a multi-tier deviceto-device communication environment [38]. In [38], the original fractional form of energy efficiency was considered as the objective function. And because of its nonconvex nature, a sub-optimal solution for channel allocation and power allocation is proposed using an iterative non-cooperative game approach. On the contrary, in this work, we transform the fractional form of energy efficiency into its subtractive which leverages to find a tractable solution using dual-multiplier Lagrangian optimization.

The following points make our investigation novel and worth investigating. First, most of the existing literature on the coexistence of LTE and WiFi focus on spectrum efficiency, while energy efficiency is largely ignored. In this study, we aim to propose an energy-efficient subchannel allocation and power allocation scheme while satisfying a) the minimum capacity requirement of dualaccess SBSs and b) the maximum tolerable interference by macro cell users and the legacy WiFi devices. Second, the interference management is also a critical issue in LTE-U based on CSAT, especially during the 'ON' period and this is far more important in the considered dense small cell environment. Third, a self-organized framework for dualaccess small cells is proposed without any involvement of a centralized entity which is an attractive option for the dense environment. Fourth, a novel less-complex iterative algorithm for the sub-channel allocation and power allocation is proposed, which is shown to converge to the Nash Equilibrium.

The rest of the paper is structured as follows: the system model and the problem formulation are presented in Section 3. The objective function transformation is presented in Section 4. The proposed self-organizing framework for the sub-channel and the power allocation is given in Section 5 and the simulation results are presented in Section 6. Finally, Section 7 concludes the paper.

\section{SYSTEM MODEL AND PROBLEM FORMULATION}

\subsection{Network model}

A network model of the dual-access small cell network is shown in Fig. 1. Considering the co-channel environment, the two main interference components that are present in the 'ON' period of LTE-U based CSAT are: cross-tier and cotier. The former one is comprised of interference between small cells and macro cells (operating in licensed bands), and between small cells and the legacy WiFi devices (operating in unlicensed bands). The later one is comprised of interference among small cells (operating in licensed and unlicensed bands). The 'ON' period of LTE-U is based on a number of WiFi characteristics including traffic load, node density, etc., and is proposed to be determined by each dual-access SBS in a self-organizing way. This overcomes the scalability problem in the centralized management of 
small cell networks. Due to the determination of ' $\mathrm{ON}^{\prime}{ }_{466}$ and 'OFF' periods at the SBS level, it becomes extremely ${ }^{467}$ important and challenging to manage the co-tier and cross- 468 tier interference components. Since the calculation of ' $\mathrm{ON}^{\prime}{ }_{469}$ and 'OFF' periods is based on the detection of WiFi signals, 470 it is possible that the received signal power of some legacy 471 WiFi devices is too low to be identified by the carrier 472 sense mechanism in CSAT [39]. Consequently, the trans- 473 mission of LTE-U in the 'ON' period can interfere with the 474 legacy WiFi devices. In order to protect and respect the 475 QoS requirement of the legacy WiFi devices, we assume 476 according to [40] that a control channel is set up over 477 the wired backhaul between the neighbouring SBSs and 478 WiFi stations. If the QoS requirement of the legacy WiFi 479 devices is violated, the concerned SBS will get an alert 480 via the control channel which switches its period from 481 'ON' to 'OFF' accordingly. In addition, we assume that the 482 legacy WiFi devices operating in unlicensed bands follow ${ }_{483}$ the LBT technique which is important for maintaining 484 a fair coexistence between LTE-U and other unlicensed 485 technologies. However, if a technology operates in unli- 486 censed bands and does not use LBT such as Bluetooth, ${ }_{487}$ the following solutions can be utilized (in any order) for 488 addressing the problem and adjusting the duty cycle of 489 LTE-U SBSs: (i) coordination of dual-access SBSs and the technology which does not employ LBT via a control channel (ii) assessment of the activity of the technology, and (iii) prediction of transmission of the technology using machine learning techniques [41] [42] [43].

In the proposed self-organizing framework, each dualaccess SBS has to perform three tasks iteratively: sensing, learning, and tuning. In the sensing task, each SBS interacts with the environment and acquire channel gains from both licensed and unlicensed bands. For determining the LTEU 'ON' or 'OFF' period, each SBS detects the WiFi transmission and adjust the duty cycle periods accordingly. In the learning task, an iterative mechanism is taken into account for the allocation of sub-channels and power levels with the concern of enhancing the energy efficiency while guaranteeing the QoS requirements of small cell users, macro cell users, and the legacy WiFi stations. In the tuning task, the best learned strategies in task 2 are updated by each dual-access SBS. The objective of the proposed selforganizing framework is to enhance the energy efficiency by exploiting sub-channels and power levels in ultra-dense small cell networks without creating harmful impact on the other network entities. In this study, we make the following assumptions:

1) Channel state information (CSI) availability: It is assumed 513 that each dual-access SBS has the capability to ac- 514 quire CSI from macro cell users (operating in licensed 515 bands), small cell users (operating in both licensed 516 and unlicensed bands), and the legacy WiFi devices 517 (operating in unlicensed band). Uplink CSI can be 518 obtained at SBSs from the uplink pilots transmitted by 519 macro cell users, small cell users, and the legacy WiFi 520 devices. In case of time division duplexing (TDD), 521 downlink CSI can be estimated due to the channel ${ }_{522}$ reciprocity between the uplink and the downlink [44]. 523 While in frequency division duplexing (FDD), due to 524 the lack of the reciprocity, estimation of downlink CSI is a difficult task and is achieved through separate downlink sounding and feedback from all the tier users. Although the CSI of the users far apart from the SBSs are hardly obtained in practice, the impact of such interferers is so small that the proposed algorithm provides an upper bound on the performance of the practical system. In order to evaluate the impact of errors in estimating the actual CSI, an analysis is also carried out with imperfect CSI.

2) Control channels for QoS satisfaction: In order to satisfy the QoS requirements of macro cell users, small cell users, and the legacy WiFi devices, we assume that reliable control channels are available that connect MBSs, SBSs and WiFi access points. In case the QoS requirements of small cell users, macro cell users, and/or the legacy WiFi devices are not satisfied, the particular SBS will be alerted from the SBS, the MBS, and/or the WiFi access point in order to change the strategies.

3) Synchronization on sub-channels: A tight synchronization is assumed among the transmitters so that the interference only occurs whenever there is a transmission on similar sub-channels.

\subsection{System model}

In this study, we consider the downlink of dual-access small cell networks with a frequency reuse- 1 in an orthogonal frequency-division multiple access (OFDMA)based two-tier cellular environment, which comprises of $K$ macro cells each operated by a MBS at its center overlaid with $N$ dual-access small cells each operated by a SBS. In addition, the frequency reuse- 1 corresponds that each dual-access SBS has complete access to licensed and unlicensed bands. The total number of $Y$ macro cell users and $X$ small cell users are randomly deployed within coverage of $K$ macro cells and $N$ small cells, respectively. Furthermore, we consider that there are $E$ legacy WiFi devices within coverage of $K$ macro cells and these are randomly deployed around the vicinity of $N$ small cells. Although we consider the duty cycle approach of CSAT for the fair LTE and WiFi coexistence, still there is an impact of interference of dual-access SBSs on the legacy WiFi devices and vice versa (both operating in unlicensed band). This interference is possible in two cases: (i) the transmission of SBSs is not detected by the legacy WiFi devices due to the hidden terminal problem and this happens because of the incompatibility of CSAT and carrier-sense multiple access (CSMA) and (ii) the transmission of the legacy WiFi devices can interfere with SBSs due to lack of LBT in LTE-U. In order to model this interference, we consider that the number of legacy WiFi access points that interfere with the dual-access SBSs is represented as $V$, where $V \subset E$.

The total number of $G$ and $H$ sub-channels are assumed to be available from licensed and unlicensed bands, respectively, and the number of sub-channels acquired by each SBS is denoted as: $Q$ and $J$, respectively, such that $Q \leq G$ and $J \leq H$, while the number of subchannels acquired by each MBS is denoted as $G$. The 
Proposed self-organizing

framework for Dual-access

small cells

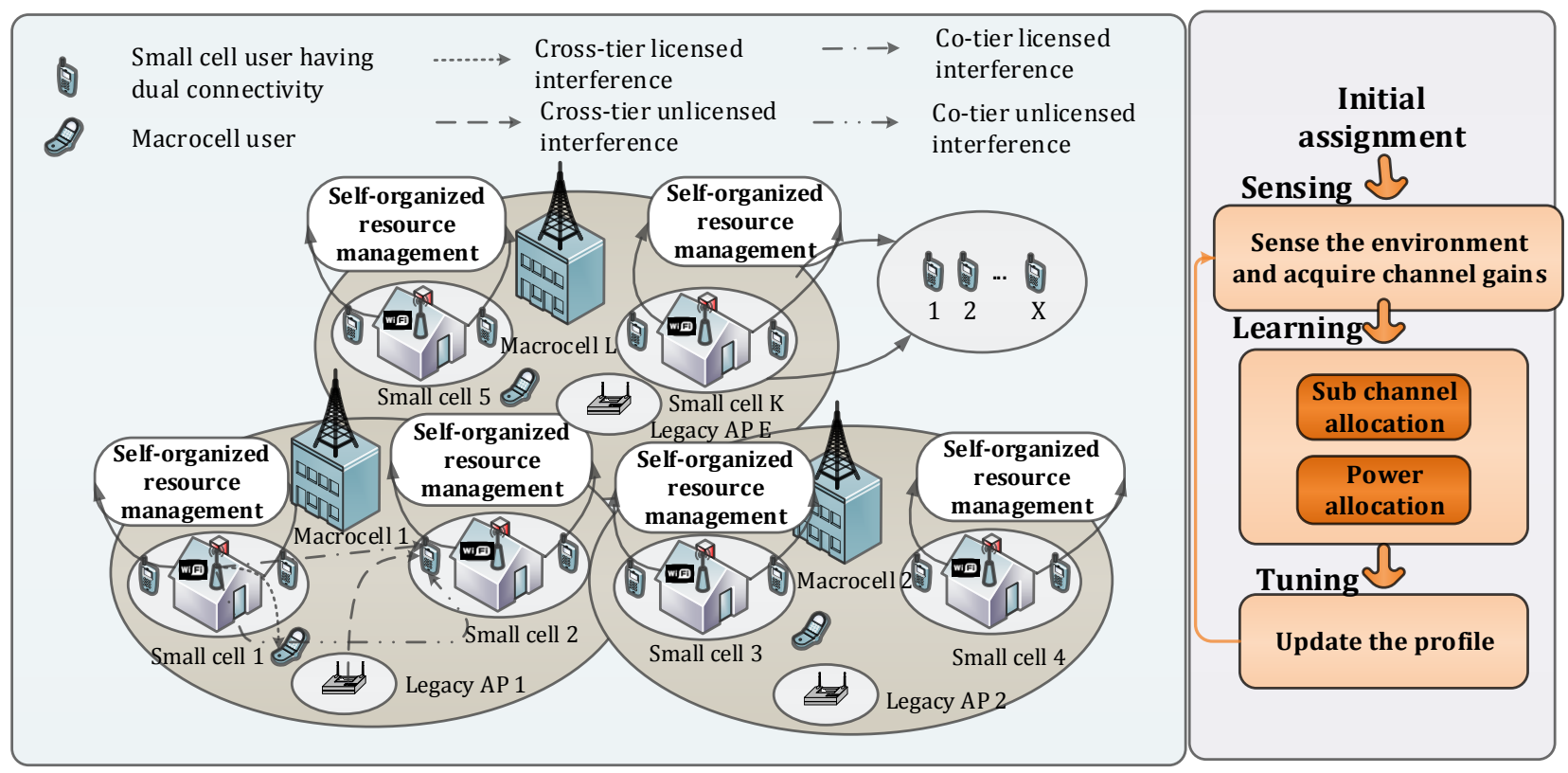

Fig. 1. Proposed self-organizing framework for ultra-dense dual-access small cell network.

total number of $R$ power levels are available on the sub- ${ }_{556}$ channels acquired by each SBS, while the power levels ${ }_{557}$ on the sub-channels acquired by MBSs are assumed to be 558 fixed. Moreover, let $G_{v}, H_{v}$ and $R_{v}$ denote the sets of sub- ${ }_{559}$ channels from licensed band, unlicensed band, and power 560 levels such that $G_{v}=\{1,2, \ldots, G\}, H_{v}=\{1,2, \ldots, H\},{ }_{561}$ and $R_{v}=\{1,2, \ldots, R\}$, respectively.

The transmission power of the $k$ th MBS in the licensed ${ }^{563}$ band sub-channels and of the $n$th dual-access SBS 564 in the licensed and unlicensed band sub-channels ${ }_{565}$ are represented as $P_{k, g}^{M} \in\left[P_{k, 1}^{M}, P_{k, 2}^{M}, \ldots, P_{k, G}^{M}\right],{ }_{566}^{565}$ $P_{n, g}^{S, L} \in\left[P_{n, 1}^{S, L}, P_{n, 2}^{S, L}, \ldots, P_{n, G}^{S, L}\right] \quad$ and $\quad P_{n, h}^{S, U L} \in{ }^{567}$ $\left[P_{n, 1}^{S, U L}, P_{n, 2}^{S, U L}, \ldots, P_{n, H}^{S, U L}\right]$, respectively, where $P_{n, g}^{S, L}=0$ if the $g$ th sub-channel from the licensed band is not acquired by the $n$th SBS; otherwise, $P_{n, g}^{S, L} \neq 0$. ${ }^{568}$ The similar argument holds for $P_{k, q}^{M}$ and $P_{n, h}^{S, U L}{ }^{569}$ The maximum power constraint on the acquired $Q{ }^{570}$ and $J$ sub-channels by MBSs and SBSs is given by $P_{M A X}^{M}$ and $P_{M A X}^{S}$ such that $\sum_{g=1}^{G} P_{k, g}^{M} \leq P_{M A X}^{M}$ and $\sum_{g=1}^{G} P_{n, q}^{S, L}+\sum_{h=1}^{H} P_{n, h}^{S, U L} \leq P_{M A X}^{S}$, respectively.

The performance of the proposed scheme is evaluated in terms of energy efficiency (expressed in bits/J) of the dual-access small cell networks. The signal-to-interference- ${ }_{577}$ plus-noise ratio (SINR) on a macro cell user associated ${ }_{578}$ with the $k$ th MBS operating on the $g$ th sub-channel is 579 given in (1) on the next page.

where $G_{k k, g}^{M \rightarrow M}, G_{a k, g}^{M \rightarrow M}$, and $G_{n k, g}^{S \rightarrow M, L}$ are the channel ${ }_{581}$ gains on the similar sub-channels between the $k$ th MBS ${ }_{582}$ and the associated macro cell user, between the $k$ th MBS ${ }_{583}$ and the $a$ th MBS, and between the $n$th SBS and the $k$ th ${ }_{584}$ MBS, respectively; $\delta_{k g}^{M}, \delta_{n g}^{S, L}, \delta_{n h}^{S, U L}$, and $\delta_{v h}^{L e g-W, U L}$ are ${ }^{585}$ the binary indicators which correspond to whether the $g$ th sub-channel from the licensed band and the $h$ th subchannel from the unlicensed band is used by the $k$ th MBS, the $n$th SBS and the $v$ th legacy WiFi access point respectively; and finally, $\sigma^{2}$ is the noise power. Similarly, the SINR on the $g$ th sub-channel from the licensed band and the $h$ th sub-channel from the unlicensed band of the $n$th SBS is given in (2) and (3) on the next page.

According to the additive modeling of error in estimating CSI as proposed in [45], here the imperfect CSI $G_{n n, g}^{S \hat{\rightarrow}, S, L}$ is modeled as the deviation from the actual CSI $G_{n n}^{S \rightarrow S, L}$ and is written as:

$$
G_{n n, g}^{S \hat{\vec{S} S, L}}=G_{n n, g}^{S \rightarrow S, L}+\Omega_{n n, g}^{S \rightarrow S, L}, \forall n .
$$

where $\Omega_{n n, g}^{S \rightarrow S, L}$ is the channel estimation error and is modeled by a zero mean Gaussian random variable with variance $\varsigma_{n n, g}^{S \rightarrow S, L}$. Additionally, the random variable $\Omega_{n n, g}^{S \rightarrow S, L}$ is independent and identically distributed (i.i.d) on $G$ and $H$ sub-channels from the licensed and unlicensed bands, respectively, and on all the tiers including small cells, macro cells, and the legacy WiFi devices. For the sake of simplicity, we consider a minimum mean square error (MMSE) channel estimator and because of that the CSI estimation error and the actual CSI become mutually uncorrelated [46]. Similarly, the imperfect CSI of the remaining channel gains in (1), (2), and (3) are modeled by (4). For the sake of simplicity, we use the actual CSI notations in the remainder of the paper. However, the corresponding imperfect CSI is introduced according to (4).

The average throughput of the $k$ th MBS operating in 


$$
S I N R_{k, g}^{M}=\frac{P_{k, g}^{M} G_{k k, g}^{M \rightarrow M}}{\sigma^{2}+\underbrace{\sum_{a=1, a \neq k}^{K} \delta_{a g}^{M} P_{a, g}^{M} G_{a k, g}^{M \rightarrow M}}_{\text {macro cell-licensed-components }}+\underbrace{\sum_{n=1}^{N} \delta_{n g}^{S, L} P_{n, g}^{S, L} G_{n k, g}^{S \rightarrow M, L}}_{\text {small cell-licensed-components }}}, \forall k, g,
$$

$$
S I N R_{n, g}^{S, L}=\frac{P_{n, g}^{S, L} G_{n n, g}^{S \rightarrow S, L}}{\sigma^{2}+\underbrace{\sum_{k=1}^{K} \delta_{k g}^{M} P_{k, g}^{M} G_{k n, g}^{M \rightarrow S}}_{\text {macro cell-licensed-components }}+\underbrace{\sum_{a=1, a \neq n}^{N} \delta_{a g}^{S, L} P_{n, g}^{S, L} G_{a n, g}^{S \rightarrow S}}_{\text {small cell-licensed-components }}}, \forall n, g,
$$

the licensed band and the $n$th SBS operating in the licensed 607 and unlicensed bands is given by,

$$
\begin{gathered}
\Psi_{k}^{M}=\frac{W}{G} \sum_{g=1}^{G} \delta_{k g}^{M} \log _{2}\left(1+S I N R_{k, g}^{M}\right), \forall k, \\
\Psi_{n}^{S, L}=\frac{W}{G} \sum_{g=1}^{G} \delta_{n g}^{S, L} \log _{2}\left(1+S I N R_{n, g}^{S, L}\right), \forall n, Q \leq G,
\end{gathered}
$$

$\Psi_{n}^{S, U L}=\frac{W}{H} \sum_{h=1}^{H} \rho_{n} \delta_{n h}^{S, U L} \log _{2}\left(1+S I N R_{n, h}^{S, U L}\right), \forall n, J \leq H_{617}^{616}$

where $W$ represents the system bandwidth, $\rho_{n}$ is the ' $\mathrm{ON}^{\prime}{ }^{619}$ period and $1-\rho_{n}$ is the 'OFF' period of the LTE-U. In the proposed dual-access framework, $\rho_{n}$ is determined by each SBS which is based on the determined WiFi transmission occupancy of dual-access SBSs and the legacy WiFi devices in unlicensed bands. In order to determine this, the dual-access SBSs are required to turn their radio frequency 625 (RF) transmission 'OFF' which can guarantee the precise 626 estimation of WiFi traffic. In addition, the sensing time ${ }_{627}$ needs to be sufficiently large to allow the SBSs to precisely ${ }_{628}$ estimate the WiFi traffic. In order to fine tune the decisions 629 regarding the 'ON' and 'OFF' periods, the received WiFi ${ }_{630}$ signal is averaged by each SBS [47].

The total average throughput of the $n$th SBS, operating 632 in both licensed and unlicensed sub-channels is given by,

$$
\Psi_{n}^{S}=\Psi_{n}^{S, L}+\Psi_{n}^{S, U L}, \forall n .
$$

Also, the energy efficiency (expressed in bits/J) of the ${ }_{637}$ $n$th SBS is given in (9) on the next page. Where $p_{c}$ is 638 the circuit power of the transmitter and is assumed to be 639 constant in this work.
Although we consider the downlink, the similar scheme can be employed for the uplink as in [48] in which CSI is estimated at SBSs from the orthogonal uplink pilots from macro cell users, small cell users and the legacy WiFi devices.

\subsection{Notations}

The notations and abbreviations used in Table 1 will be used throughout the rest of the paper.

\subsection{Problem formulation}

Since the goal of the study is to enhance the energy efficiency of the dual-access small cell network by exploiting sub-channel allocation and power allocation from licensed and unlicensed bands, the problem P1 is formulated along with the system constraints and is given in (10) and (11) on the next page. Where $G_{k n, q, z}^{S \rightarrow M, L}$ is the channel gain between the $n$th SBS and the $z$ th macro user of the $k$ th MBS operating on the $g$ th sub-channel. The similar description holds for $G_{v n, h, e}^{S \rightarrow L e g-W L}$, but between the $n$th SBS and $n$th legacy WiFi device. The binary indicators $\delta_{n g}^{S, L}$ and $\delta_{n h}^{S, U L}$ are the output optimization variables and are included in P1 in the form of $A_{n}^{S}$, while $\delta_{k g}^{M}$ and $\delta_{v h}^{L e g-W, U L}$ are the binary input indicators and are updated randomly.

The constraint $\mathrm{C} 1$ refers to the minimum capacity requirement of dual-access SBSs and is represented as $R_{M I N}^{S}$. The value is proposed to be selected by the operator of SBSs. If the constraint is violated, then the SBS gets an alert from the MBS to change the strategies so that the minimum capacity requirement can be achieved. The constraint $\mathrm{C} 2$ is used to protect macro cell users from dual-access SBSs operating in licensed bands. For this, the maximum tolerable limit is selected and is represented as $\zeta^{M, L}$ which can be adjusted by the MBS operator based on the location of users in the coverage area. In case the constraint C2 is violated, the concerned SBS gets an alert 


$$
E E_{n}^{S}=\frac{\frac{W}{G} \sum_{g=1}^{G} \delta_{n g}^{S, L} \log _{2}\left(1+S I N R_{n, g}^{S, L}\right)+\frac{W}{H} \sum_{h=1}^{H} \rho_{n} \delta_{n h}^{S, U L} \log _{2}\left(1+S I N R_{n, h}^{S, U L}\right)}{\sum_{g=1}^{G} \delta_{n g}^{S, L}\left(P_{n, g}^{S, L}+p_{c}\right)+\sum_{h=1}^{H} \rho_{n} \delta^{S, U L}\left(P_{n, h}^{S, U L}+p_{c}\right)}, \forall n .
$$

TABLE 1

Notation and assumptions.

\begin{tabular}{|c|c|}
\hline Parameters & Meaning \\
\hline MBS & Macro cell base station; \\
\hline SBS & Dual-access small cell base station; \\
\hline$K$ & Number of macro cells; \\
\hline$N$ & Number of small cells; \\
\hline$E$ & Number of Legacy WiFi APs; \\
\hline$V$ & Number of Legacy WiFi APs that are outside the sensing range of SBS; \\
\hline$X$ & Number of macro cell users; \\
\hline$Y$ & Number of small cell users; \\
\hline$k$ & Index for macro cell; \\
\hline$n$ & Index for dual-access small cell; \\
\hline$e$ & Index for Legacy WiFi access point; \\
\hline$G$ & Total pool of sub-channels from licensed band; \\
\hline$Q$ & Number of sub-channels from licensed band acquired by each SBS at a particular time; \\
\hline$H$ & Total pool of sub-channels from unlicensed band; \\
\hline$J$ & Number of sub-channels from unlicensed band acquired by each SBS at a particular time; \\
\hline$G_{v}$ & Set of sub-channels from licensed band; \\
\hline$H_{v}$ & Set of sub-channels from unlicensed band; \\
\hline$W$ & System bandwidth; \\
\hline$R$ & Transmission power levels; \\
\hline$R_{v}$ & Set of transmission power levels; \\
\hline$L$ & Indicator for licensed band; \\
\hline$U L$ & Indicator for unlicensed band; \\
\hline$P_{n, q}^{S, L}$ & Transmission power of the $n$th SBS on the $g$ th sub-channel from licensed band; \\
\hline$P_{n, h}^{S, U L}$ & Transmission power of the $n$th SBS on the $h$ th sub-channel from unlicensed band; \\
\hline$P_{k, g}^{M}$ & Transmission power of the $k$ th MBS on the $g$ th sub-channel from licensed band; \\
\hline$G_{n k, g}^{S \stackrel{k, g}{\rightarrow}, L}$ & Perfect channel gain between the $n$th SBS and the $k$ th MBS operating on the $g$ th sub-channel from licensed band; \\
\hline$G_{n \rightarrow, g}^{S} \underset{\sim}{\rightarrow} S, L$ & Imperfect channel gain between the $n$th SBS and the associated small cell user operating on the $g$ th sub-channel from licensed band; \\
\hline$\Omega_{n n, g}^{S \rightarrow S}, L$ & Channel estimation error between the $n$th SBS and the associated small cell user operating on the $g$ th sub-channel from licensed band; \\
\hline$\varsigma_{n n, g}^{S \rightarrow S, L}$ & Variance of the channel estimation error between the $n$th SBS and the associated small cell user operating on the $g$ th sub-channel from licensed band; \\
\hline$\delta_{k g}^{M}$ & Binary indicator which indicates whether the $g$ th sub-channel from licensed band is used by the $k$ th MBS; \\
\hline$\rho_{n}$ & 'ON' period of SBSs; \\
\hline$\Psi_{n}^{S, L}$ & Average throughput of the $n$th SBS operating on licensed band sub-channels; \\
\hline$\Psi_{n}^{S, U L}$ & Average throughput of the $n$th SBS operating on unlicensed band sub-channels; \\
\hline$A_{n}^{S}$ & Sub-channel allocation vector of the $n$th SBS; \\
\hline$P_{n}^{S}$ & Power allocation vector of the $n$th SBS; \\
\hline$\theta_{n}^{n}$ & Strategy profile associated with the $n$th SBS; \\
\hline$U_{n}$ & Utility function associated with the $n$th SBS; \\
\hline$A_{n}^{* S}$ & Optimal sub-channel allocation vector of the $n$th SBS; \\
\hline$P_{n}^{* S}$ & Optimal power allocation of the $n$th SBS; \\
\hline$\zeta^{M, L}$ & Maximum interference incurred by SBSs in licensed sub-channels that a MBS can tolerate; \\
\hline$\kappa^{L e g-W, U L}$ & Maximum interference incurred by SBSs in unlicensed sub-channels that a legacy WiFi device can tolerate; \\
\hline$R_{M I N}^{S}$ & Minimum capacity requirement of dual-access SBSs; \\
\hline$\eta_{n}^{* S^{*}}$ & Maximum energy efficiency of the $n$th SBS; \\
\hline$U_{n}$ & Utility function of the $n$th SBS; \\
\hline
\end{tabular}

via the established control channels that connects SBSs 657 and MBSs. Similarly, to protect the legacy WiFi devices, 658 the maximum allowed interference limit represented as 659 $\kappa^{L e g-W, U L}$ is used in the constraint C3. Again, if the inter- 660 ference exceeds the limit, the culprit WiFi device sends an 661 alert to the SBS to change the strategies via the established 662 control channels between WiFi access points and SBSs. The 663 constraints $\mathrm{C} 4$ and $\mathrm{C} 5$ correspond to the selection of $G$ and 664 $H$ sub-channels from the pool of $Q$ and $J$ sub-channels 665 from licensed and unlicensed bands, respectively. The 666 constraint C6 imposes the maximum power transmission 667 limit of $P_{M A X}^{S}$ on SBSs while selecting the power levels on 668 the $G$ and $H$ sub-channels.

\section{OBJECTIVE FUNCTION TRANSFORMATION} The assignment variables $\delta_{n g}^{S, L}$ and $\delta_{n h}^{S, U L}$ for selecting 672 the sub-channels from licensed and unlicensed bands in 673
P1 make the problem a combinatorial programming one, which is NP-hard [49]. The fractional form objective function in (9) generally is not a concave function but by assuming certain conditions on the constraints, the function can fall into the class of it [50]. Thus, the objective function in (9) is non-convex and traditional convex optimization techniques cannot be applied for finding the optimal solution. The only option left is to employ a brute force method but the complexity of such a method increases exponentially with sub-channels and powers levels, which is not suitable even for a small network. Therefore, we aim to propose an efficient and a less-complex solution for such a challenging problem.

In order to do so, we transform the problem P1 in (10) using a non-linear fractional integer programming approach [51]. Then, we develop an effective sub-channel allocation and power allocation scheme for solving the en- 


$$
\begin{aligned}
& P 1:\left(A_{n}^{* S}, P_{n}^{* S}\right)
\end{aligned}
$$

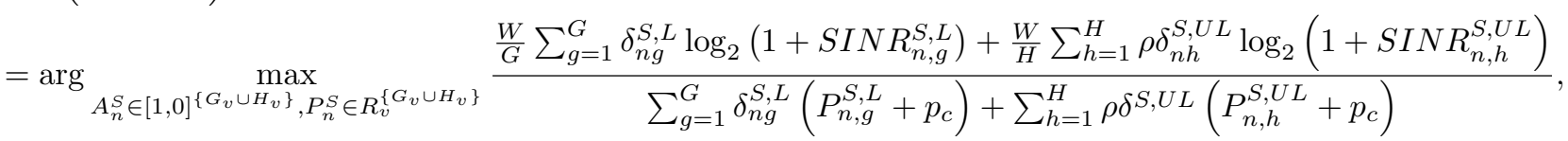

$$
\begin{aligned}
& C 1: \Psi_{n}^{S, L}+\Psi_{n}^{S, U L} \geq R_{M I N}^{S}, \forall n, \\
& C 2: \sum_{g=1}^{G} P_{n, g}^{S, L} G_{k n, g, z}^{S \rightarrow M, L} \delta_{n g}^{S, L} \leq \zeta^{M, L}, \forall k, z, n, \\
& C 3: \sum_{h=1}^{H} P_{n, h}^{S, U L} G_{v n, h, e}^{S \rightarrow L e g-W, U L} \delta_{n h}^{S, U L} \leq \kappa^{L e g-W, U L}, \forall v, e, n, \\
& C 4: \sum_{g=1}^{G} \delta_{n g}^{S, L} \leq Q, \forall n \\
& C 5: \sum_{h=1}^{H} \delta_{n h}^{S, U L} \leq J, \forall n \\
& C 6: \sum_{g=1}^{G} \delta_{n g}^{S, L} P_{n, g}^{S, L}+\sum_{h=1}^{H} \delta_{n h}^{S, L} P_{n, h}^{S, U L} \leq P_{M A X}^{S} .
\end{aligned}
$$

ergy efficiency maximization problem. Since the objective function in P1 has a non-linear fractional programming form [51], we can define the maximum energy efficiency $\eta_{n}^{* S}$ as:

$$
\eta_{n}^{* S}=\frac{\phi_{\text {num }}\left(A_{n}^{* S}, P_{n}^{* S}\right)}{\phi_{\text {den }}\left(P_{n}^{* S}\right)},
$$

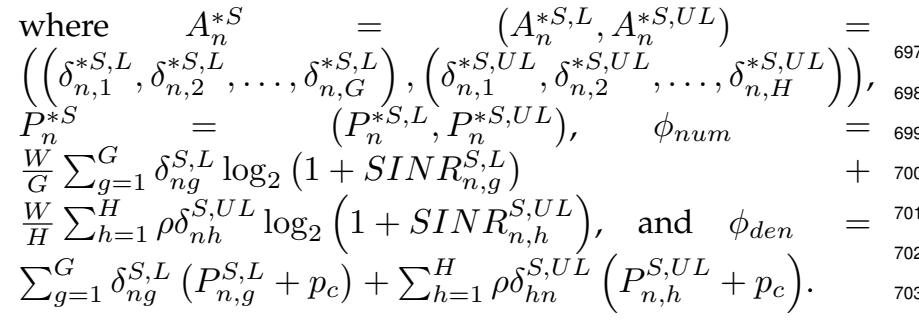

Theorem 1. The maximum energy efficiency $\eta_{n}^{* S}$ is ${ }_{706}$ achieved if the condition in (13) on the next page is 707 satisfied. A.

Proof: The proof of Theorem 1 is given in Appendix ${ }_{709}$

Thus, Theorem 1 reveals that for any objective function, which is in a fractional form, there exists an equivalent objective function in a subtractive form e.g., $\phi_{\text {num }}\left(A_{n}^{S}, P_{n}^{S}\right)-$ $\eta_{n}^{* S} \phi_{\text {den }}\left(P_{n}^{S}\right)$ in our case. However, the optimal energy efficiency $\eta_{n}^{* S}$ in (12) cannot be achieved directly and in 716 order to obtain that, we propose an iterative Algorithm 1717 in Section 5. Therefore, the problem P1 can be transformed 718 into its equivalent problem $\mathbf{P} \mathbf{2}$ and is represented as:

$$
\begin{aligned}
& P 2:\left(A_{n}^{* S}, P_{n}^{* S}\right)= \\
& \max _{A_{n}^{S} \in[1,0]^{\left\{G_{v} \cup H_{v}\right\}}, P_{n}^{S} \in R_{v}^{\left\{G_{v} \cup H_{v}\right\}}} \phi_{\text {num }}\left(A_{n}^{S}, P_{n}^{S}\right)- \\
& \eta_{n}^{* S} \phi_{\text {den }}\left(P_{n}^{S}\right),
\end{aligned}
$$

subject to: $\mathrm{C} 1$ - C6.

The transformed problem $\mathbf{P 2}$ in (14) is in a subtractive form and constitutes a tractable and a feasible solution. In order to develop an effective algorithm for solving the problem P2, we can define the following function $F\left(\eta_{n}^{S}\right)=$

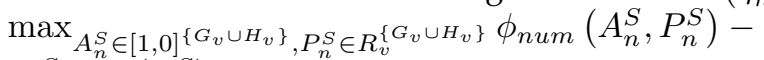
$\eta_{n}^{* S} \phi_{\text {den }}\left(P_{n}^{S}\right)$ with Lemma 1 given below.

Lemma 1. For all feasible $A_{n}^{S}, P_{n}^{S}$, and $\eta_{n}^{S}, F\left(\eta_{n}^{S}\right)$ is a strictly monotonic decreasing function in $\eta_{n}^{S}$, and $F\left(\eta_{n}^{S}\right) \geq 0$.

Proof: The proof of Lemma 1 is given in Appendix B.

In the remainder of the paper, we use the transformed problem $\mathbf{P 2}$.

\section{NON-COOPERATIVE GAME FORMULATION FOR SUB-CHANNEL ALLOCATION AND POWER ALLOCA- TION}

\subsection{Non-cooperative game}

A non-cooperative game is a powerful mathematical tool and is used for a variety of resource allocation problems in wireless networks [52]. In the problem formulation in Section 3 and its transformation in Section 4, we consider 


$$
\max _{A_{n}^{S} \in[1,0]^{\left\{G_{v} \cup H_{v}\right\}}, P_{n}^{S} \in R_{v}^{\left\{G_{v} \cup H_{v}\right\}}} \phi_{\text {num }}\left(A_{n}^{S}, P_{n}^{S}\right)-\eta_{n}^{* S} \phi_{\text {den }}\left(P_{n}^{S}\right)=\phi_{\text {num }}\left(A_{n}^{* S}, P_{n}^{* S}\right)-\eta_{n}^{* S} \phi_{\text {den }}\left(P_{n}^{* S}\right)=0 .
$$

that each dual-access SBS is a selfish and an irrational player, which tends to increase its utility function. Therefore, the problem P1 presented in (10) and its equivalent subtractive transformed form $\mathbf{P 2}$ in (14) can be modeled as a non-cooperative game. In this study, we model the joint sub-channel allocation and power allocation for dualaccess small cells via a non-cooperative game. Specifically, this joint allocation is represented by a normal form game such that $\Gamma=\left\{N, \theta_{n}, U_{n}\right\}$, where $N$ is the finite number of players, $\theta_{n}$ is the strategy associated with each player, and $U_{n}$ is the utility function which associates the reward to each player. Mathematically, the utility function can be thought of as a mapping function that maps the strategy $\theta_{n}$ into a real number $\Re$ such that $U_{n}: \theta_{n} \rightarrow \Re$. In addition, the strategies of the other players involved in the game is represented by $\theta_{-n}=\left\{\theta_{1}, \theta_{2}, \ldots, \theta_{n-1}, \theta_{n+1}, \ldots, \theta_{N}\right\}$.

In the proposed non-cooperative game, a set of $N$ dualaccess SBSs are the players of the game, which interact with the environment in a self-organizing manner and select the strategies $\theta_{n}$ : the sub-channels and the power levels. Mathematically, the strategy of the game is represented as $\theta_{n}=\left\{A_{n}^{S}, P_{n}^{S}\right\}$, where $A_{n}^{S}=\left\{A_{n}^{S, L}, A_{n}^{S, U L}\right\}$ is 774 an indication vector that corresponds to the allocation of 775 the $Q$ and $J$ sub-channels from the total pool of $G$ and 776 $H$ sub-channels, respectively, while $P_{n}^{S}=\left\{P_{n}^{S, L}, P_{n}^{S, U L}\right\}$ refers to the power levels on the selected sub-channels from both licensed and unlicensed bands. For a given $A_{n}^{S}$ and $P_{n}^{S}$, the strategies of the other players can be represented as $A_{-n}^{S}=\left\{A_{1}^{S}, A_{2}^{S}, \ldots, A_{n-1}^{S}, A_{n+1}^{S}, \ldots, A_{N}^{S}\right\}$ and $P_{-n}^{S}=\left\{P_{1}^{S}, P_{2}^{S}, \ldots, P_{n-1}^{S}, P_{n+1}^{S}, \ldots, P_{N}^{S}\right\}$, respectively. 778 Since the goal of the proposed non-cooperative game is 779 to enhance the energy efficiency, the utility function is 780 considered as the transformed form problem P2 and is 781 written as:

$$
U_{n}\left(A_{n}^{S}, P_{n}^{S}\right)=\phi_{\text {num }}\left(A_{n}^{S}, P_{n}^{S}\right)-\eta_{n}^{* S} \phi_{\text {den }}\left(P_{n}^{S}\right) .
$$

Definition 1. For a given $A_{n}^{S}$ and $P_{n}^{S}$, the best response dynamics of the joint sub-channel allocation and power allocation of the $n$th dual access SBS is written in (16) on the next page.

The joint sub-channel allocation and power allocation in the transformed form in $\mathbf{P 2}$ is a mixed integer non-linear problem which is generally intractable in its original form [53]. In order to provide an efficient and a less-complex solution, we follow the traditional approach [54], [55], and solve the problem P2 in two steps: sub-channel allocation and power allocation.

\subsection{Sub-channel allocation}

In the sub-channel allocation step, the following Theorem provides the optimal sub-channel allocation for the given power levels computed in the previous iteration. Here, 785 $N$ SBSs select $G$ and $J$ sub-channels from licensed and 786 unlicensed bands, respectively, from (17).
Theorem 2. A group of $Q$ and $J$ sub-channels from the pool of $G$ and $H$ sub-channels in licensed and unlicensed bands can only be allocated to the $n$th dual-access SBS, if the following condition is satisfied:

$$
\begin{array}{r}
\left(A_{n}^{* S}\right)=\arg \min _{\left.A_{n}^{S} \in[1,0]\right]^{\left\{G_{v} \cup H_{v}\right\}}}\left(\sum_{g=1}^{G} \delta_{n g}^{S, L} \frac{I_{n, g}^{S, L}}{G_{n n, g}^{S \rightarrow, L}}\right. \\
\left.+\sum_{h=1}^{H} \rho \delta_{n h}^{S, U L} \frac{I_{n, h}^{S, U L}}{G_{n n, h}^{S \rightarrow S, U L}}\right),
\end{array}
$$

where $I_{n, g}^{S, L}=\sigma^{2}+\sum_{k=1}^{K} \delta_{k g}^{M} P_{k, g}^{M} G_{k n, g}^{M \rightarrow S}+$ $\sum_{a=1, a \neq n}^{N} \delta_{a g}^{S, L} P_{a, g}^{S, L} G_{a n, g}^{S \rightarrow, L} \quad$ and $\quad I_{n, h}^{S, U L}=$ $\sigma^{2}+\sum_{a=1, a \neq n}^{N} \delta_{a h}^{S, U L} P_{a, h}^{S, L} G_{a n, h}^{S \rightarrow S, U L}$

Proof: Given the power allocation of the $n$th player, the best response of the sub-channel allocation is represented as:

$$
\left(A_{n}^{* S}\right)=\arg \max _{A_{n}^{S} \in[1,0]^{\left\{G_{v} \cup H_{v}\right\}}} U_{n}\left(A_{n}^{S} \mid P_{-n}^{S}\right),
$$

\section{s.t.C4 and C5}

Since the utility function is transformed from (9) to (15), the sub-channel allocation represented in (17) can be written as:

$$
\left(A_{n}^{* S}\right)=\arg \max _{\left.A_{n}^{S} \in[1,0]^{\{G v} \cup H_{v}\right\}} \phi_{\text {num }}\left(A_{n}^{S}, P_{n}^{S}\right)-\eta_{n}^{* S} \phi_{\text {den }}\left(P_{n}^{S}\right) .
$$

In order to prove the condition (17) in Theorem 2, we put (2), (3), $I_{n, g}^{S, L}, I_{n, h}^{S, U L}$, and the definitions from (12) $\left(\phi_{\text {num }}\left(A_{n}^{S}, P_{n}^{S}\right), \eta_{n}^{* S}, \phi_{\text {den }}\left(P_{n}^{S}\right)\right)$ in (19) and a new representation of the sub-channel allocation can be formed in (20) which is given on the next page.

Assuming $\omega_{1}$ and $\omega_{2}$ to be the target SINR on the selected sub-channels from licensed and unlicensed bands and substituting the power levels of the corresponding SBS $P_{n, g}^{S, L}=\frac{\omega_{1} I_{n, g}^{S, L}}{G_{n n, g}^{S, S, L}}$ and $P_{n, h}^{S, U L}=\frac{\omega_{2} I_{n, h}^{S, U L}}{G_{n n, h}^{S S, U L}}$, we get a new sub-channel allocation representation (21) given on the next page. Furthermore, we found that the subchannel allocation representation in (21) is equivalent to the expression below and hence, Theorem 2 is proved.

$$
\begin{aligned}
& \left(A_{n}^{* S}\right)=\arg \min _{A_{n}^{S} \in[1,0]^{\left\{G_{v} \cup H_{v}\right\}}}\left(\sum_{g=1}^{G} \delta_{n g}^{S, L} \frac{I_{n, g}^{S, L}}{G_{n n, g}^{S \rightarrow S, L}}\right. \\
& \left.+\sum_{h=1}^{H} \rho \delta_{n h}^{S, U L} \frac{I_{n, h}^{S, U L}}{G_{n n, h}^{S \rightarrow S, U L}}\right) .
\end{aligned}
$$

\subsection{Power allocation}

Given the sub-channel allocation of each SBS, the power allocation problem can be modeled as a non-cooperative power optimization game such that $\Gamma^{\prime}=\left\{N, P_{n}^{S}, U_{n}\right\}$. 


$$
\left(A_{n}^{* S}, P_{n}^{* S}\right)=\max _{A_{n}^{S} \in[1,0]^{\left\{G_{v} \cup H_{v}\right\}}, P_{n}^{S} \in R_{v}^{\left\{G v \cup H_{v}\right\}}} \phi_{n u m}\left(A_{n}^{S}, P_{n}^{S}\right)-\eta_{n}^{* S} \phi_{\text {den }}\left(P_{n}^{S}\right) .
$$



$$
\begin{aligned}
& -\eta_{n}^{* S}\left(\sum_{g=1}^{G} \delta_{n g}^{S, L} P_{n, g}^{S, L}+\sum_{h=1}^{H} \rho \delta_{n h}^{S, U L} P_{n, h}^{S, U L}+p_{c}(Q+\rho J)\right) .
\end{aligned}
$$

$$
\begin{aligned}
\left(A_{n}^{* S}\right)=\arg \max _{A_{n}^{S} \in[1,0]^{\left\{G_{v} \cup H_{v}\right\}}} \frac{W}{G} \sum_{g=1}^{G} \delta_{n g}^{S, L} \log _{2}\left(1+\omega_{1}\right)+\frac{W}{H} \sum_{h=1}^{H} \rho \delta_{n h}^{S, U L} \log _{2}\left(1+\omega_{2}\right) \\
\\
\quad-\eta_{n}^{* S}\left(\omega_{1} \sum_{g=1}^{G} \delta_{n g}^{S, L} \frac{I_{n, g}^{S, L}}{G_{n n, g}^{S \rightarrow S, L}}+\omega_{2} \sum_{h=1}^{H} \rho \delta_{n h}^{S, U L} \frac{I_{n, h}^{S, U L}}{G_{n n, h}^{S \rightarrow S, U L}}+p_{c}(Q+\rho J)\right) .
\end{aligned}
$$

Since the utility function of the proposed scheme depends 819 upon the behavior (power levels) of two or more players, 820 we model the power optimization problem via a non- 821 cooperative power optimization game [56]. For the given 822 sub-channel allocation, the best response dynamics of the $n$th player is represented as

$$
\left(P_{n}^{* S}\right)=\arg \max _{P_{n}^{S} \in R_{v}^{\left\{G_{v} \cup H_{v}\right\}}} U_{n}\left(P_{n}^{S} \mid P_{-n}^{S}\right),
$$

\section{s.t.C1 - C6}

The players involved in the non-cooperative power optimization game optimize their power levels independently on $Q$ and $J$ sub-channels. Since the goal of the optimization problem is to enhance the energy efficiency of dual-access small cells without creating harmful impact on small cell users, macro cell users, and the legacy WiFi devices, the power optimization is carried out within the network constraints $\mathrm{C} 1$ - C6.

For a given sub-channel allocation, the utility function in subtractive form is a convex function of $P_{n}^{S}$ and the power level strategy is also convex. Thus, the KarushKuhn-Tucker (KKT) condition can be applied to find out the optimal solution.

The Lagrangian associated with the transformed problem P2 in (14) is written in (24) on the next page, where $\alpha_{n}, \beta_{n}, \gamma_{n}$, and $\chi_{n}$ are the Lagrangian multipliers associated with the constraints $\mathrm{C} 1, \mathrm{C} 2, \mathrm{C} 3$, and $\mathrm{C} 6$, respectively.

The equivalent dual problem can be decomposed into two sub problems: the maximization problem solves the power allocation on the selected sub-channels and the minimization problem solves for the Langrangian multipliers which is given by,

$\min _{\alpha_{n} \geq 0, \beta_{n} \geq 0, \gamma_{n} \geq 0 \chi_{n} \geq 0} \max _{P_{n}^{S} \in R_{v}^{\left\{G_{v} \cup H_{v}\right\}}} \lambda_{E E}\left(P_{n}^{S}, \alpha_{n}, \beta_{n}, \gamma_{n}, \chi_{n}\right) \stackrel{825}{826}_{824}^{825}$

For a given energy efficiency $\eta_{n}^{S}$, the solution is given 828 in (26) on the next page,

The Lagrangian multipliers $\alpha_{n}, \beta_{n}, \gamma_{n}$, and $\chi_{n}$ are updated according to the gradient method and are given below,

$\alpha_{n}(\tau+1)=\left[\alpha_{n}(\tau)-\mu_{m, \beta}(\tau)\left(\nabla_{n}^{S, L}+\nabla_{n}^{S, U L}-R_{M I N}^{S}\right)\right]$.

$$
\begin{aligned}
\beta_{n}(\tau+1)= & {\left[\beta_{n}(\tau)-\mu_{n, \gamma}(\tau)\left(\zeta^{M, L}\right.\right.} \\
& \left.\left.-\sum_{g=1}^{G} P_{n, g}^{S, L} G_{k n, g, z}^{S \rightarrow M, L} \delta_{n g}^{S, L}\right)\right] .
\end{aligned}
$$

$$
\begin{array}{r}
\gamma_{n}(\tau+1)=\left[\gamma_{n}(\tau)-\mu_{\gamma}(\tau)\left(\kappa^{\text {Leg }-W, U L}\right.\right. \\
\left.\left.-\sum_{h=1}^{H} P_{n, h}^{S, U L} G_{e, b, n}^{S \rightarrow \text { Leg }-W, U L} \delta_{n h}^{S, U L}\right)\right]
\end{array}
$$

$$
\begin{aligned}
\chi_{n}(\tau+1) & =\left[\chi_{n}(\tau)-\mu_{\chi}(\tau)\left(P_{M A X}^{S}\right.\right. \\
& \left.\left.-\sum_{g=1}^{G} \delta_{n g}^{S, L} P_{n, g}^{S, L}+\sum_{n h}^{S, L} P_{n, h}^{S, U L}\right)\right] .
\end{aligned}
$$

\subsection{Proposed iterative algorithm}

The proposed iterative algorithm employs two steps: the sub-channel allocation and the power allocation. Based on Lemma 1, an iterative algorithm is proposed to solve the transformed problem $\mathbf{P} 2$ by updating $\eta_{n}^{(i), F}$ in each iteration. In addition, the proposed algorithm ensures that 


$$
\begin{gathered}
\lambda_{E E}\left(P_{n}^{S}, \alpha_{n}, \beta_{n}, \gamma_{n}\right)=\Phi_{n u m}\left(A_{n}^{S}, P_{n}^{S}\right)-\eta_{n}^{S} \phi_{\operatorname{den}}\left(P_{n}^{S}\right)+\alpha_{n}\left(\Psi_{n}^{S, L}+\Psi_{n}^{S, U L}-R_{M I N}^{S}\right)+\beta_{n}\left(\zeta^{M, L}-\sum_{g=1}^{G} P_{n, g}^{S, L} G_{k n, g, z}^{S \rightarrow M, L} \delta_{n g}^{S, L}\right) \\
+\gamma_{n}\left(\kappa^{L e g-W, U L}-\sum_{h=1}^{H} P_{n, h}^{S, U L} G_{e, b, n}^{S \rightarrow L e g-W, U L} \delta_{n h}^{S, U L}\right)+\chi_{n}\left(P_{M A X}^{S}-\sum_{g=1}^{G} \delta_{n g}^{S, L} P_{n, g}^{S, L}+\sum_{h=1}^{H} \delta_{n h}^{S, L} P_{n, h}^{S, U L}\right), \quad(24)
\end{gathered}
$$

$$
\begin{aligned}
& P_{n}^{* S}=\left(P_{n, g}^{* S, L}, P_{n, h}^{* S, U L}\right) \\
& P_{n}^{S}=\left(\left[\delta_{g n}^{S, L} \frac{\frac{W}{Q}\left(1+\alpha_{n}\right) \log _{2} e}{\eta_{n}^{S}+\beta_{n} G_{k n, g, z}^{S \rightarrow M, L}+\chi_{n}}-\delta_{g n}^{S, L} \frac{\sigma^{2}+\sum_{k=1}^{K} P_{k, g}^{M} G_{k n, g}^{M \rightarrow S} \delta_{k g}^{M}+\sum_{a=1, a \leq n}^{N} G_{a n, g}^{S \rightarrow S, L} \delta_{a g}^{S, L}}{G_{n n, g}^{S \rightarrow S, L}}\right]^{+}\right. \\
& \left.\left[\delta_{h n}^{S, U L} \frac{\frac{W}{J}\left(1+\alpha_{n}\right) \log _{2} e}{\eta_{n}^{S}+\gamma_{n} G_{e, b, n}^{S \rightarrow L e g-W, U L}+\chi_{n}}-\delta_{h n}^{S, U L} \frac{\sigma^{2}+\sum_{a=1, a \leq n}^{N} P_{a, h}^{S, U L} G_{a n, h}^{S \rightarrow S, U L} \delta_{a h}^{S, U L}}{G_{n n, h}^{S \rightarrow S, U L}}\right]^{+}\right] .
\end{aligned}
$$

$\eta_{n}^{(i), F}$ increases in each iteration and gives the optimal 864 energy efficiency performance. Further, it can be seen that 865 the proposed iterative algorithm comprises of two steps: 866 sub-channel allocation and power allocation. In the sub- 867 channel allocation step, the allocation of $Q$ and $J$ sub- 868 channels from the total pool of $G$ and $H$ sub-channels 869 in licensed and unlicensed bands, respectively, is carried 870 out by each dual-access small cells with the concern of 871 minimizing the impact of interference. In the power allo- 872 cation step, the power levels on the selected sub-channels 873 are evaluated via a non-cooperative power optimization game under the constraints C1 - C6. In addition, in each ${ }_{875}$ iteration of the iterative algorithm the parameter $\eta_{n}^{(i), F}$ is ${ }_{876}^{875}$ updated until the optimality is achieved.

The following Theorem 3 and Theorem 4 elaborate the ${ }_{878}$ existence of a Nash Equilibrium point and the convergence 879 to the optimal energy efficiency, respectively.

Theorem 3. A Nash Equilibrium point exists in the pro- 881 posed non-cooperative power optimization game $\Gamma^{\prime}=882$ $\left\{N, P_{n}^{S}, U_{n}\right\}$. The optimal strategy set of power allo- ${ }^{883}$ cation $\left\{\left(P_{n, g}^{* S, L}, P_{n, h}^{* S, U L}\right) \mid n \in N, g \in G, h \in H\right\}^{884}$ which is achieved by applying Algorithm 1 is the Nash ${ }^{885}$ Equilibrium.

C.

Proof: The proof of Theorem 3 is given in Appendix

Theorem 4. The proposed iterative Algorithm 1 converges to the optimal energy efficiency $\eta_{n}^{* S}, \forall n \in N$.

Proof: The proof of Theorem 4 Appendix D.

\subsection{Implementation issues and complexity analysis}

In this section, the implementation issues and complexity 896 analysis of the proposed scheme are discussed. In the ${ }_{897}$ proposed self-organizing framework, each SBS only needs 898 to estimate the received interference rather than the precise 899 sub-channel allocation and power allocation strategies of 900 other players. According to (17) and (22), the required 901 information can be obtained by the estimated interference. Since we consider the downlink, each SBS can acquire CSI from its associated small cell users which estimate the downlink channels gains of all the interferers including $K$ MBSs, $N-1$ SBSs, and $V$ legacy WiFi access points. In TDD based systems, downlink CSI can be estimated due to the channel reciprocity between uplink and downlink. However, in FDD based systems, downlink CSI is possible by extra downlink sounding and feedback from users [44]. The channel gains $G_{n n, g}^{S \rightarrow S, L}, G_{k n, g}^{M \rightarrow S}, G_{a n, g}^{S \rightarrow S, L}, G_{n n, h}^{S \rightarrow S, U L}$, and $G_{v n, h}^{L e g-W \rightarrow S, U L}$ are required in the sub-channel allocation and the power allocation steps. These channel gains can be estimated by measuring the downlink channel gains on $G$ licensed and $H$ unlicensed channels from MBSs (operating in licensed channels only), SBSs (operating in licensed and unlicensed channels), and the legacy WiFi devices (operating in unlicensed channels only) from small cell users and pass them to the concerned SBS. In real systems, it can be hard to precisely estimate CSI and for this, we carried out an analysis of the proposed scheme with imperfect CSI. Thus, Algorithm-1 can be implemented in each dual-access SBS by utilizing local information and have little interaction with the MBS.

We analyse and compare the computational complexity of the proposed scheme (Algorithm-1) with the RR-NPOG scheme in a multi small cellular environment. The details of RR-NPOG are given in Section 6. For the sub-channel allocation step, the computation complexity can be represented as $O(N(G+H))$. Given $\Delta_{i}$ is the maximum number of iterations for the power allocation step, the computational complexity can be represented as $O\left(\Delta_{i} N\right)$. Therefore, given $\Delta_{0}$ is the maximum number of iterations for the iterative sub-channel allocation and power allocation scheme, the over computational complexity of Algorithm1 is $O\left(\Delta_{0}\left(N(G+H)+\Delta_{i} N\right)\right)$. It is noted that $\Delta_{0}$ and $\Delta_{i}$ are the maximum number of iterations of the proposed iterative and power allocation steps, respectively. However, within the maximum iteration counts, the convergence 


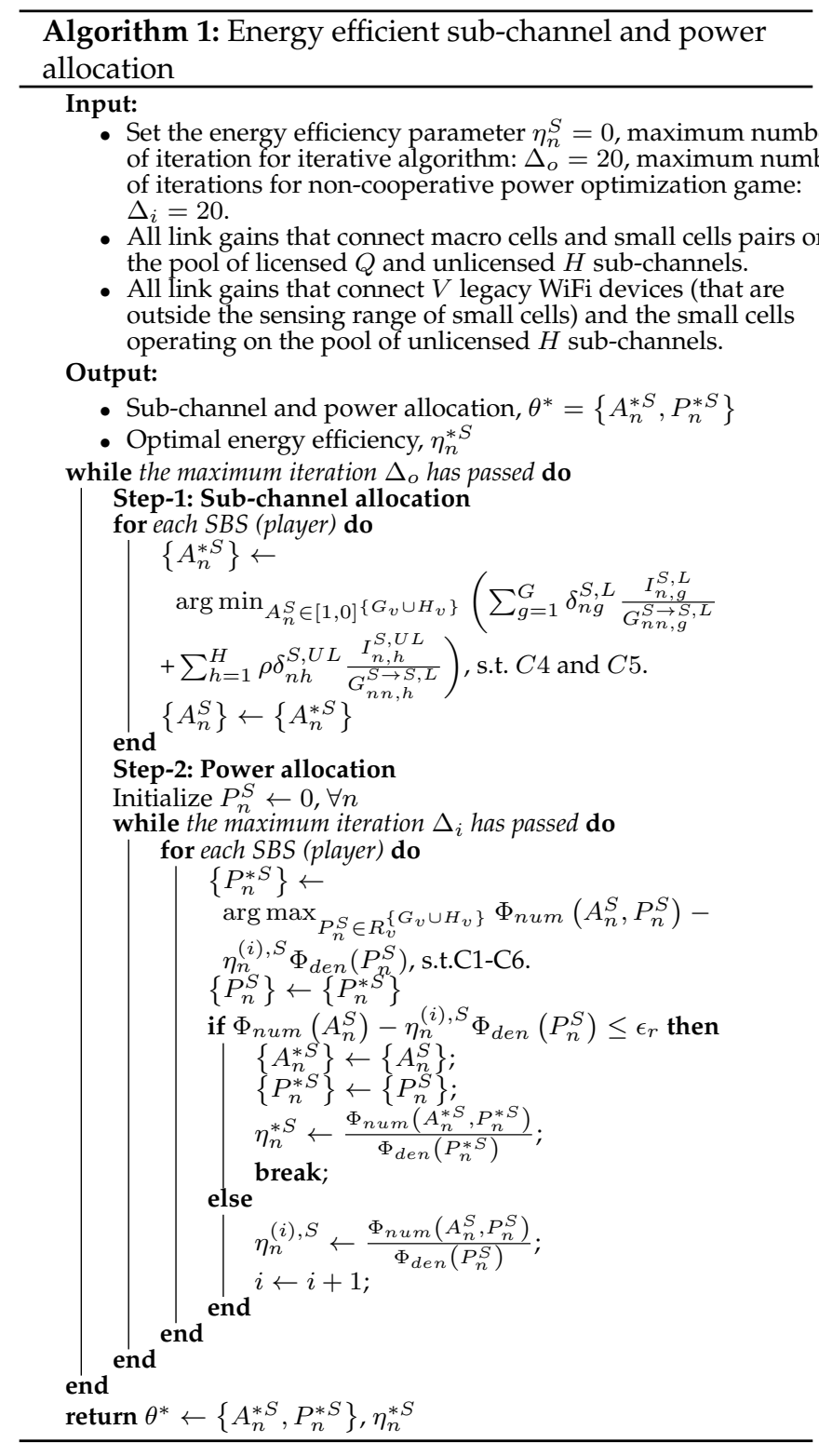

can be achieved if there is no further improvement in 920 the energy efficiency and the computational complexity 921 is lesser than $O\left(\Delta_{0}\left(N(G+H)+\Delta_{i} N\right)\right)$. The computa- 922 tional complexity of the proposed scheme without using 923 Lagrangian optimization in the power allocation step is 924 written as $O\left(\Delta_{0}\left(N(G+H)+\Delta_{i} N R\right)\right)$ which justifies the 925 usage of optimization in the power allocation step. The 926 computational complexity of RR-NPOG is represented as 927 $O\left(\Delta_{0} N\left(1+\Delta_{i}\right)\right)$. Although the computational complexity 928 of the RR-NPOG is lower compared to the proposed ${ }_{929}$ scheme, the energy efficiency performance of our scheme 930 is significantly higher. This is discussed in detail in Sec- ${ }_{931}$ tions 6.2 - 6.4 .

\section{Simulation RESUlts}

In this section, the energy efficiency performance of the 975 proposed self-organizing framework is evaluated in terms 976 of: 1) the convergence; 2) the energy efficiency distri- 977 bution; 3) the impact of varying sub-channels, $G, H, Q$, 978 and $J$; 4) the impact of dual-access small cell density, $N$; and 5) the impact of legacy WiFi access points, $V$. In addition, the spectrum efficiency performance of the proposed scheme and RR-NPOG is also evaluated with respect to the small cell density. In addition, in each of the analysis, the proposed scheme is evaluated with perfect and imperfect CSI such that 'Proposed scheme (perfect CSI)' and 'Proposed scheme (imperfect CSI)'. The inclusion of this imperfectness in CSI is to analyze the degradation of the proposed scheme when CSI is not precisely known. For the sake of comparison, two self-organizing schemes using round-robin non-cooperative power optimization (labelled as RR-NPOG) and a spectrum efficiency game (labelled as spectrum efficient) are utilized which can be considered as the representatives schemes.

The goal of the RR-NPOG scheme is to enhance the energy efficiency which is similar to the proposed scheme. However, it utilizes a round robin (RR) mechanism as the sub-channel allocation step and uses the similar power optimization game approach. The concern of comparing the proposed scheme with RR-NPOG is to show the importance of jointly optimizing sub-channels and power levels. The goal of the spectrum efficient scheme is to enhance the spectrum efficiency instead of the energy efficiency. The reason for this comparison is to investigate how much energy efficiency performance is archived if the proposed game is aligned with spectrum efficiency and not with energy efficiency.

\subsection{Simulation setup}

We consider the downlink of OFDMA-based two-tier cellular networks operating in the carrier frequency of $2 \mathrm{GHz}$ which closely follows the 3GPP TR 25.814 specifications. Further, for the sake of simplicity, a single macro cellular environment $(K=1)$ is considered with a radius of $1000 \mathrm{~m}$. In addition, there are $N$ small cells underlaid within the macro cell each with a radius of $40 \mathrm{~m}$. Each MBS and SBS is located at the center of the corresponding cell and $X$ and $Y$ are the macro cell users and the small cell users, respectively, and are randomly deployed within a coverage area of each cell. In order to illustrate the impact of the legacy WiFi devices on the small cells that are outside their sensing range, different values of $V$ are considered such that $V \in\{5,10,15,20\}$

The total pool of licensed and unlicensed bands is divided into $G$ and $H$ sub-channels, respectively, where each sub-channel has a bandwidth of $180 \mathrm{KHz}$. In order to evaluate the efficiency of the proposition, different pool of licensed and unlicensed sub-channels are also considered such that $G \in\{10,20\}$ and $H \in\{10,20\}$. Further, the total number of sub-channels acquired by each SBS is considered to be $Q \in\{2,4,5,6,8\}$ and $J \in\{2,4,5,6,8\}$ from licensed band and unlicensed bands, respectively, while the number of sub-channels acquired by each MBS is assumed to be fixed at $G=10$ and 20, respectively. The maximum transmission power constraint on MBS and SBS operating on either licensed or unlicensed subchannels is set as $P_{M A X}^{M}=43 \mathrm{dBm}$ and $P_{M A X}^{S}=23 \mathrm{dBm}$, respectively. Also, in total $R=100$ power levels are considered that are uniformly distributed in the range 
from -100 to $23 \mathrm{dBm}$. Finally, a constant circuit power of $p_{c}=100 \mathrm{~mW}$ is considered (similarly as in [57]) and the noise spectral density is set to $N_{0}=-141 \mathrm{dBW} / \mathrm{Hz}$. For modeling the imperfectness in estimating CSI, the variance of the estimation error $\varsigma_{n n, g}^{S \rightarrow S, L}$ was considered as 0.02 and 0.2 , which illustrates the minimum and maximum CSI impairment impact, respectively, on the energy efficiency and the spectrum efficiency [58].

The pathloss models (PL) that we are utilizing in this study are in accordance with the 3GPP specification [59], [60] and can be expressed as:

- For macro cell and small cell (outdoor): $P L(d B)=$ $127+30 \log _{10}(d)$

- For macro cell and small cell (indoor): $P L(d B)=$ $128.1+37.6 \log _{10}(d)$

The log normal shadowing with the shadowing factors of $8 \mathrm{~dB}$ and $4 \mathrm{~dB}$ are utilized for indoor and outdoor environment, respectively. The performance of the proposed scheme against the counterparts are evaluated for 1000 different realization of the network and the average results in terms of the network realizations and the number of involved $N$ SBSs are presented. The simulation of the proposed scheme along with the comparative counter parts including the RR-NPOG, spectrum-efficient games, ${ }^{1038}$ and the channel modeling were carried out in MATLAB.

In the remainder of the sub-sections, the following 1040 naming conventions are used for the proposed scheme ${ }^{1041}$ (with perfect and imperfect CSI) such that 'Proposed ${ }^{1042}$ scheme (perfect CSI) L and UL: $G=20, H=20, Q=5$, 1043 $J=5^{\prime}$, 'Proposed scheme (imperfect CSI) L and UL: ${ }^{1044}$ $G=20, H=20, Q=5, J=5^{\prime}$, and 'Proposed scheme ${ }^{1045}$ L and UL: $G=20$ and $H=20^{\prime}$. For instance, 'Proposed ${ }^{1046}$ scheme (perfect CSI) L and UL: $G=20, H=20, Q=5$, ${ }^{1047}$ $J=5^{\prime}$ means that the proposed scheme (with perfect CSI) ${ }^{1048}$ in which each SBS acquires $Q=5$ sub-channels from the ${ }^{1049}$ pool of $G=20$ and $J=5$ sub-channels from the pool of ${ }^{1050}$ $H=20$ licensed and unlicensed bands, respectively. Sim- ${ }^{1051}$ ilarly, the other naming conventions such as 'RR-NPOG ${ }^{1052}$ $\mathrm{L}$ and UL: $G=10, H=10, Q=5, J=5^{\prime}$ ', 'spectrum ${ }^{1053}$ efficient $\mathrm{L}$ and UL: $G=10, H=10, Q=5, J=5^{\prime}$, etc. can be described in the same way. It is important to note that the perfect CSI is considered when it is not categorically mentioned in the result figures or in their description.

\subsection{Convergence analysis}

The energy efficiency convergence analysis of the pro- 1059 posed scheme along with the comparative counterparts 1060 for two difference scenarios $N=20$ and 40 is shown 1061 in Fig. 2. The energy efficiency of the proposed scheme 1062 and the comparative counterpart schemes are determined 1063 from (9). The unavailability of precise CSI in the proposed 1064 scheme (with imperfect CSI) reduces the energy efficiency 1065 performance to $5 \%$ and $8 \%$ as compared to the proposed 1066 scheme (with perfect CSI) when the small cell density 1067 increases from $N=20$ to 40 , respectively. Although all 1068 the considered schemes converge to the optimal points for 1069 both the scenarios $(N=20$ and 40$)$ in similar number of 1070 iterations, the spectrum efficient scheme performs worst 1071 even as compared to RR-NPOG, and this is because the 1072 objective function in the spectrum efficient scheme is not 1073

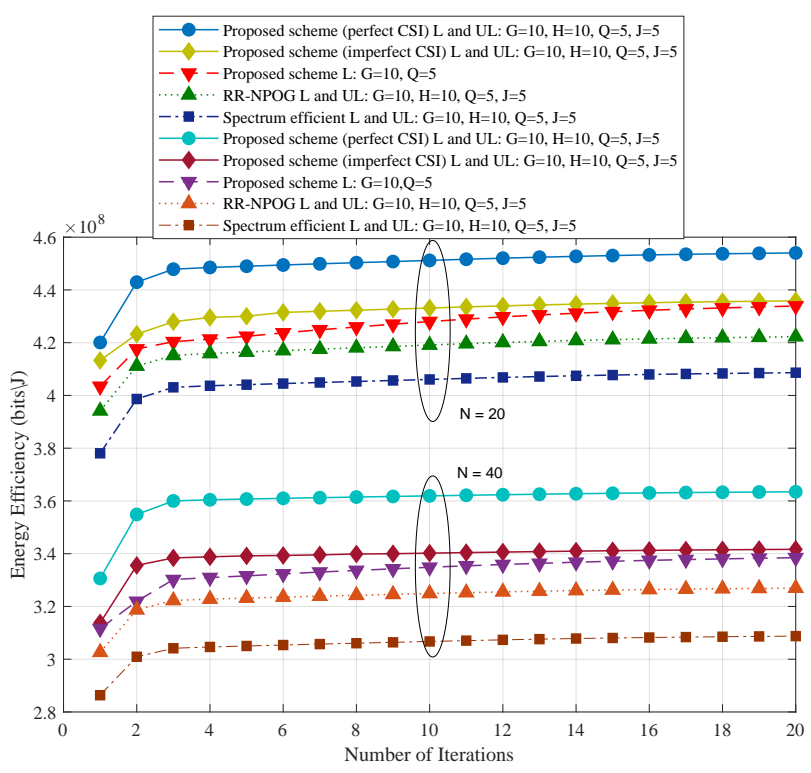

Fig. 2. Convergence performance: $V=5$.

aligned with energy efficiency. In addition, the comparison of the proposed scheme employing both licensed and unlicensed band with the one employing only licensed band reveals the importance of exploiting unlicensed band without creating harmful impact on other small cell users, the macro cell users and the legacy WiFi users. Moreover, it can also be concluded that for an energy efficient design, the power allocation using only the round robin subchannel as in RR-NPOG is not sufficient. On the other hand, although the energy efficiency decreases with the increase of small cell density, the improvement of the proposed scheme in relation to the comparative ones (proposed scheme operating on the licensed band, RRNPOG and spectrum efficient) in the listed order increases from $5 \%, 7.6 \%$ and $10.9 \%$ to $7.73 \%, 11.04 \%$ and $17.6 \%$, respectively, for the same number of iterations.

\subsection{Energy efficiency distribution}

The cumulative distribution function (CDF) of the energy efficiency of the proposed scheme along with the counterparts for 1000 different network realizations, where each realization is averaged over the involved SBSs is shown in Fig. 3. The convergence point for each realization was considered fixed at the 20th game iteration because after that there was no performance improvement of the proposed scheme and the counterparts. In addition, the results are plotted for two different small cell network densities $N=20$ and $N=40$. It can be seen that the performance trend of the proposed scheme and the comparative counter parts remain the same as in the convergence analysis plot. Similarly, The performance trend of the proposed scheme with perfect and imperfect CSI remains similar as in the convergence analysis result. The spectrum efficient scheme performs worst throughout the distribution which illustrates the importance of exploiting energy efficiency. In addition, the reason of incorporating unlicensed bands while comparing the proposed scheme operating on licensed 


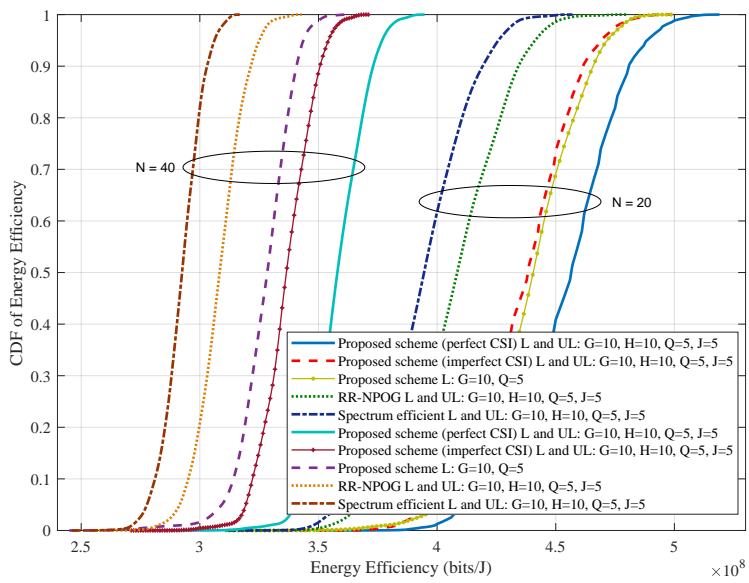

Fig. 3. Energy efficiency distribution: $V=5$.

bands only reveals the benefit of aggregation without creating harmful impact on other network entities. Also, it can be evident that the optimized power allocation scheme with round-robin sub-channel allocation performs inefficiently and the joint approach of sub-channel allocation and power allocation provides a graceful improvement in the energy efficiency throughout the whole distribution. Specifically, the min-max energy bounds which is defined as $5 \%$ to $95 \%$ of the energy efficiency of the proposed scheme operating in licensed and unlicensed bands, proposed scheme operating on licensed bands, RR-NPOG and spectrum efficient are $\left[3.55 .91 \times 10^{8}\right],\left[3.124 .988 \times 10^{8}\right]$, $\left[3.104 .80 \times 10^{8}\right]$ and $\left[3.11-4.57 \times 10^{8}\right]$ for $N=20$ and $\left[2.88-3.94 \times 10^{8}\right],\left[2.453 .63 \times 10^{8}\right],\left[2.533 .42 \times 10^{8}\right]$ and $\left[2.463 .16 \times 10^{8}\right]$ for $N=40$, respectively.

\subsection{Energy efficiency and spectrum efficiency}

Fig. 4 illustrates the results of the proposed scheme in 1125 terms of spectrum efficiency and energy efficiency (both ${ }_{1126}$ for perfect CSI and imperfect CSI with $\varsigma=0.02$ and ${ }_{1127}$ 0.2 ) with respect to the transmit power of SBSs and for ${ }_{1128}$ different small cell densities $N=20$ and 40. It can be ${ }_{1129}$ seen that the energy efficiency increases for all the density 1130 and the CSI cases, and then goes down as the transmit power increases from -100 to $23 \mathrm{dBm}$, while the spectrum efficiency increases. The goal of the proposed scheme is to maximize the energy efficiency while meeting the minimum capacity requirement of SBSs indicated by $R_{M I N}^{S} \cdot{ }_{1132}$ We considered the maximum energy efficiency point as 1133 $10 \%$ less than the maximum point and the corresponding 1134 spectrum efficiency point $R_{M I N}^{S} / W$ (bits $/ \mathrm{s} / \mathrm{Hz}$ ) as shown 1135 by the vertical line in Fig. 4. The reason for this selection 1136 is that there is a significant rise in the spectrum efficiency ${ }_{1137}$ as the energy efficiency reduces from the maximum point 1138 to $10 \%$. However, the system operating point (energy effi- ${ }_{1139}$ ciency and spectrum efficiency) can be selected anywhere 1140 in the region indicated by the double arrow in Fig. 4. ${ }^{1141}$ Because after the crossing of the energy efficiency and 1142 spectrum efficiency curves there is a negligible increase in 1143 the spectrum efficiency but there is a huge drop in the ${ }_{1144}$ energy efficiency. In order to quantify the imperfection 1145 in CSI estimation, the following two variance values are 1146

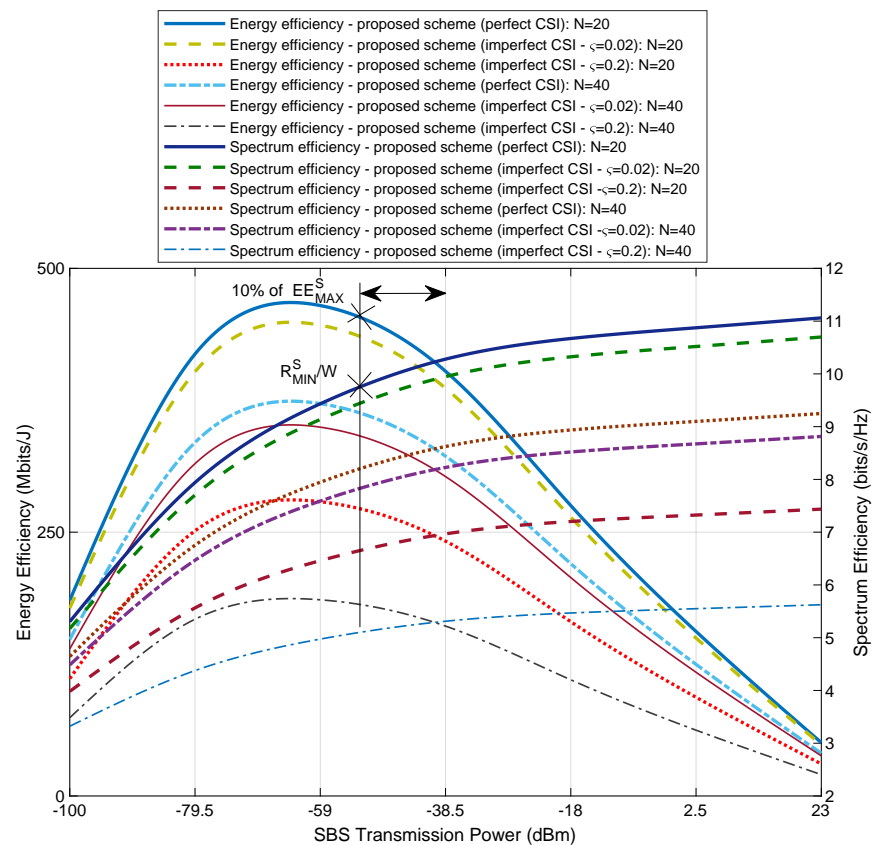

Fig. 4. Energy efficiency and spectrum efficiency for perfect and imperfect CSI: $N=20$ and $N=40$.

taken into account such as $\varsigma=0.02$ and 0.2 . It is noted that as the estimation error $\varsigma$ increases from 0.02 to 0.2 , there is a drop of $5.5 \%$ and $33.3 \%$ in the energy efficiency for the small cell density $N=20$. It can be noted that as long as CSI error is small (e.g., $\varsigma=0.02$ ), the maximum performance degradation of the proposed scheme (with imperfect CSI) is reasonable small (5.5\%) as compared to the perfect CSI. Furthermore, the energy efficiency and the spectrum efficiency of the proposed scheme with perfect CSI each drops to $25 \%$ when the small cell density increases from $N=20$ to $N=40$. The reason is that the increase of the small cell density and the fixed pool of shared sub-channels ( $G$ and $H$ ), the spectrum efficiency decreases due to the increase in the number of interference components in SINR (according to (1) and (2)) and thus, the energy efficiency also decreases.

\subsection{Impact of sub-channels $\mathrm{G}, \mathrm{Q}, \mathrm{H}, \mathrm{J}$}

The impact of varying $G, Q, H$, and $J$ on the energy efficiency of the proposed scheme and the comparative counterparts is shown in Fig. 5(a) and Fig. 5(b), for $N=20$ and $N=40$, respectively. It is clear that for the similar pool of sub-channels $G$ and $H$ and varying the sub-channels $Q$ and $J$ which are acquired by SBSs, the energy efficiency of the proposed scheme (perfect and imperfect CSI) increases at a faster rate as compared to the other comparative counter parts and this is due to the exploitation of the joint sub-channel allocation and power allocation. In addition, while comparing the results of $G=\{25,50\}$ and $N=20$ with $G=\{25,50\}$ and $N=40$ it is evident that the performance trend becomes more significant with the increase of small cell density which further illustrates the efficiency of the proposition. 


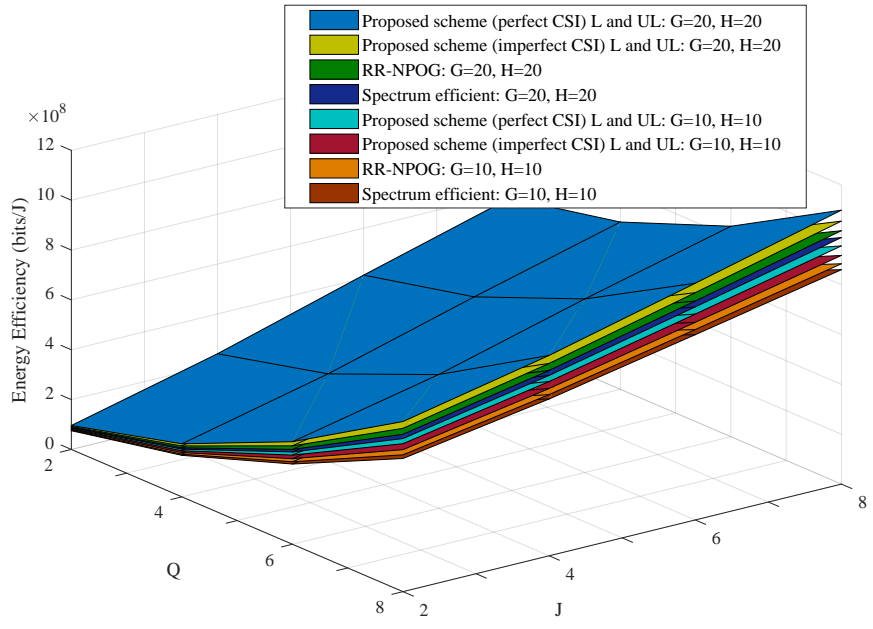

(a)

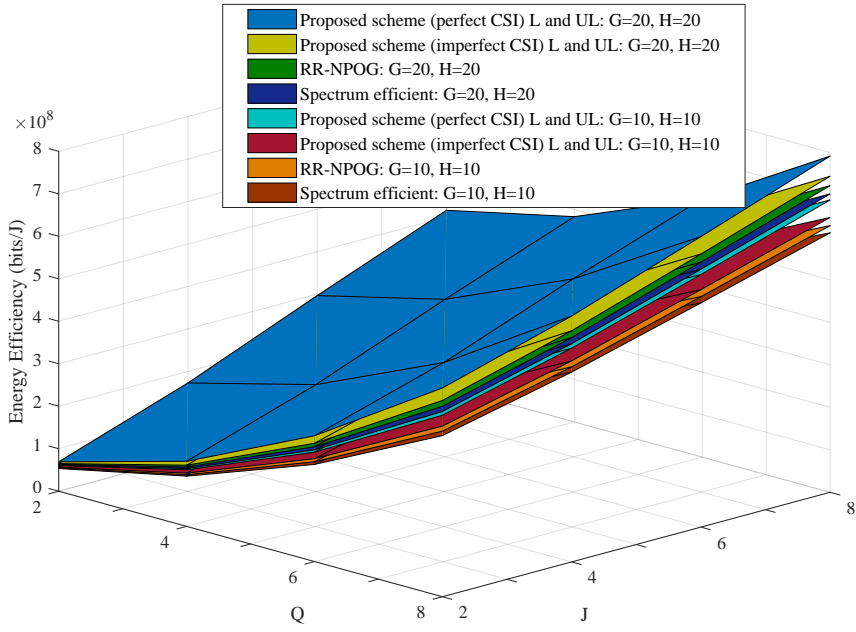

(b)

Fig. 5. Impact of varying $Q$ and $J$ on the energy efficiency: $V=5$ (a): $N=20$ and (b) $N=40$.

\subsection{Impact of small cell density}

The impact of varying the small cell density on the energy efficiency and the spectrum efficiency of the proposed schemes and the comparative counter parts is shown in Fig. 6 and 7, respectively. It is noted that due to the cochannel deployment, increasing the pool of sub-channels $\{G, H\}$ from $\{10,10\}$ to $\{20,20\}$ provides more opportunities for managing the interference and thus, enhances the energy efficiency and the spectrum efficiency. Moreover, as the sub-channel utilization increases (by increasing $N$ or decreasing $G$ and $H$ ), the proposed scheme in terms of spectrum efficiency and energy efficiency shows more graceful degradation compared to the counterpart schemes. Both the energy efficiency and the spectrum efficiency shows the similar decaying trend, the reason is that the goal of the optimization problem $\mathbf{P 1}$ is to enhance or achieve $10 \%$ of the maximum energy efficiency while satisfying the minimum SBSs capacity requirement represented as $R_{M I N}^{S}$ and the maximum tolerable interference by macro cell users and the legacy WiFi users represented as $\zeta^{M, L}$ and $\kappa^{L e g-W, U L}$, respectively, as represented in Fig. 4.

\subsection{Impact of legacy WiFi access points}

Since the determination of 'ON' and 'OFF' duty cycle is proposed to be done at the SBS level based on the detection of the WiFi signals, it is not always possible to correctly detect the presence of WiFi signals, especially if they are outside the sensing range of small cells. In order to see the impact of the such legacy WiFi access points on the energy efficiency, we are considering different densities of the legacy WiFi access points such that $V \in\{5,10,15,20\}$ and is shown in Fig.8 . It can be seen as the density of the legacy WiFi access points increases, the energy efficiency 1184 reduces and this is due to the cross-tier interference (be- 1185 tween the WiFi access points and the dual-access small 1186 cells) operating in similar unlicensed sub-channels. The 1187 decaying trend of the energy efficiency with respect to 1188

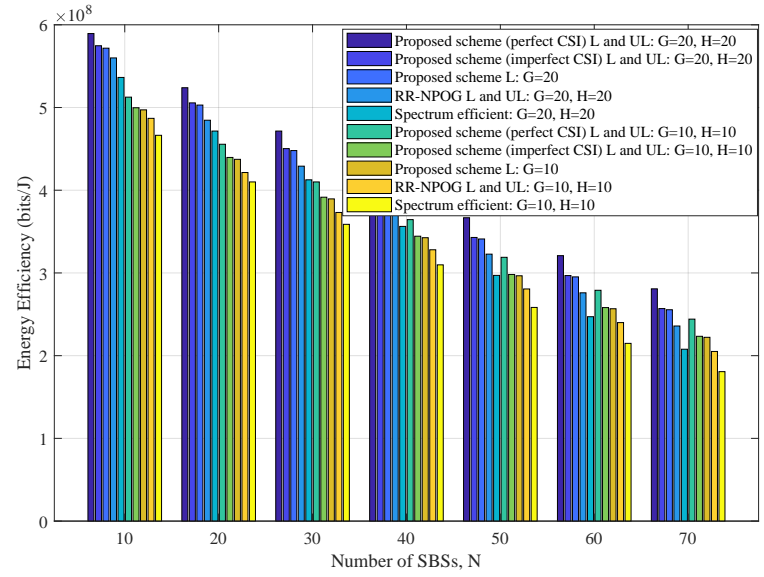

Fig. 6. Impact of small cell density on energy efficiency: $V=5$.



Fig. 7. Impact of small cell density on spectrum efficiency: $V=5$.

the WiFi density is more prominent when the dual-access density $N$ changes from 20 to 40 . The reason being that on one hand the resource utilization increases (due to increase of small cells in the co-channel environment) but on the other hand, the impact of cross-tier interference also 


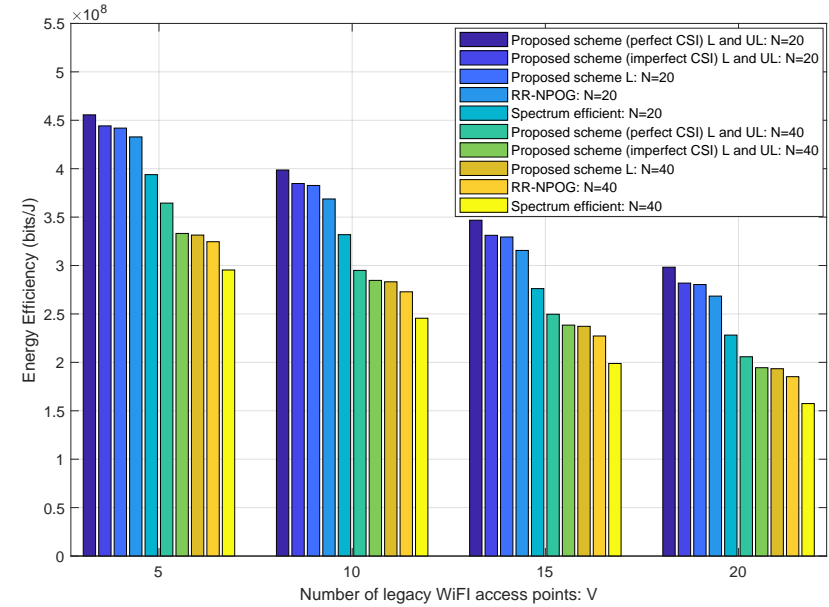

Fig. 8. Impact of legacy WiFi access points: $G=10, Q=5, H=10^{1243}$ and $J=5$. increases (due to increase of WiFi access points).

\section{CONCLUSION}

In this study, a self-organizing framework for dual-access ${ }_{1253}^{1252}$ small (operating on both licensed and unlicensed bands) ${ }^{1254}$ is presented aiming to enhance their energy efficiency per- ${ }^{1255}$ formance by exploiting sub-channel allocation and power ${ }_{1257}^{1256}$ allocation without creating harmful impact on other net- 1258 work entities. More precisely, the proposed scheme miti- 1259 gates the cross-tier and co-tier interference in the 'ON' pe- ${ }_{1261}^{1260}$ riod of dual-access small cells based CSAT. The proposed ${ }_{1262}$ scheme is designed via a non-cooperative game, where 1263 each dual-access small cell is acting as the player and the ${ }^{1264}$ sub-channels and the power levels are the strategies asso- ${ }_{1266}^{1265}$ ciated with each game. Since the goal of the proposition is 1267 to enhance the energy efficiency, the utility function of the ${ }^{1268}$ proposed game is designed in a manner that is aligned ${ }^{1269}$ not only with the energy efficiency but also the cross- 1271 tier and co-tier interference components, respectively. Fur- 1272 thermore, the fractional form objective function of the ${ }^{1273}$ energy efficiency is transformed into a subtract form by a ${ }_{1275}^{1274}$ linear fractional programming approach which is proven 1276 to provide the convergence to the Nash Equilibrium. the ${ }^{1277}$ other hand, in order to reduce the complexity of the pro- ${ }_{1279}$ posed game using joint sub-channel allocation and power ${ }_{1280}$ allocation, the joint approach is divided into two individ- 1281 ual problems: the sub-channel allocation and the power ${ }^{1282}$ allocation. Furthermore, the performance of the proposed ${ }^{1283}$ scheme is compared not only with the conventional RR- ${ }_{1285}$ NPOG and spectrum efficient schemes but also with the 1286 proposed scheme operating on only licensed band. The ${ }_{1288}^{1287}$ performance of the proposed scheme is also evaluated in ${ }_{1289}$ terms of perfect and imperfect CSI. The performance gap 1290 of the proposed scheme makes it attractive for dense small ${ }^{1291}$ cell environments.

\section{ACKNOWLEDGMENTS}

The research leading to these results has received the ${ }^{1297}$ funding from the Agency for Innovation by Science and ${ }_{1299}^{1298}$ Technology, Flanders (IWT-V) (under Grant agreement No. 1300
IWT140048 SAMURAI Project [www.samurai-project.be]) and from the European Horizon 2020 Programme (under Grant agreement No. 688116 eWINE Project [www.ewineproject.eu]).

\section{REFERENCES}

[1] A. R. Elsherif, W.-P. Chen, A. Ito, and Z. Ding, "Resource allocation and inter-cell interference management for dual-access small cells," IEEE Journal on Selected Areas in Communications, vol. 33, no. 6, pp. 1082-1096, 2015.

[2] R. Taori and A. Sridharan, "Point-to-multipoint in-band mmwave backhaul for $5 \mathrm{~g}$ networks," IEEE Communications Magazine, vol. 53, no. 1, pp. 195-201, 2015.

[3] B. Bangerter, S. Talwar, R. Arefi, and K. Stewart, "Networks and devices for the $5 \mathrm{~g}$ era," IEEE Communications Magazine, vol. 52 , no. 2, pp. 90-96, 2014.

[4] C.-X. Wang, F. Haider, X. Gao, X.-H. You, Y. Yang, D. Yuan, H. Aggoune, H. Haas, S. Fletcher, and E. Hepsaydir, "Cellular architecture and key technologies for $5 \mathrm{~g}$ wireless communication networks," IEEE Communications Magazine, vol. 52, no. 2, pp. 122-130, 2014

[5] Z. Zhou, M. Dong, K. Ota, and Z. Chang, "Energy-efficient context-aware matching for resource allocation in ultra-dense small cells," IEEE Access, vol. 3, pp. 1849-1860, 2015.

[6] R. Zhang, Z. Zheng, M. Wang, X. Shen, and L.-L. Xie, "Equivalent capacity in carrier aggregation-based lte-a systems: A probabilistic analysis," IEEE Transactions on Wireless Communications, vol. 13, no. 11, pp. 6444-6460, 2014.

[7] Qualcomm, "1000x:more small cells, hyper-dense small cell deployments," Available at http://www.qualcomm.com.

[8] C. Yang, J. Li, and M. Guizani, "Cooperation for spectral and energy efficiency in ultra-dense small cell networks," IEEE Wireless Communications, vol. 23, no. 1, pp. 64-71, 2016.

[9] H. Zhang, S. Chen, X. Li, H. Ji, and X. Du, "Interference management for heterogeneous networks with spectral efficiency improvement," IEEE Wireless Communications, vol. 22, no. 2, pp. 101-107, 2015.

[10] Y. Wang, H. Ji, and H. Zhang, "Spectrum-efficiency enhancement in small cell networks with biasing cell association and eicic: An analytical framework," International Journal of Communication Systems, vol. 29, no. 2, pp. 362-377, 2016.

[11] A. Shahid, S. Aslam, H. S. Kim, and K.-G. Lee, "A docitive qlearning approach towards joint resource allocation and power control in self-organised femtocell networks," Transactions on Emerging Telecommunications Technologies, vol. 26, no. 2, pp. 216230, 2015

[12] G. Y. Li, Z. Xu, C. Xiong, C. Yang, S. Zhang, Y. Chen, and S. Xu, "Energy-efficient wireless communications: tutorial, survey, and open issues," IEEE Wireless Communications, vol. 18, no. 6, 2011.

[13] R. Q. Hu and Y. Qian, "An energy efficient and spectrum efficient wireless heterogeneous network framework for $5 \mathrm{~g}$ systems," IEEE Communications Magazine, vol. 52, no. 5, pp. 94-101, 2014.

[14] S. Dimatteo, P. Hui, B. Han, and V. O. Li, "Cellular traffic offloading through wifi networks," in Mobile Adhoc and Sensor Systems (MASS), 2011 IEEE 8th International Conference on. IEEE, 2011, pp. 192-201.

[15] Lte-u forum. Available at http://www.lteforum.org/

[16] 3gpp release 13 specification, 2015. http://www.3gpp.org/rele$13 /$.

[17] "Ite-u technical report: coexistence study for lte-u sdl version 1.0," Available at http://lteuforum.org/ documents.html.

[18] A. M. Cavalcante, E. Almeida, R. D. Vieira, S. Choudhury, E. Tuomaala, K. Doppler, F. Chaves, R. C. Paiva, and F. Abinader, "Performance evaluation of lte and wi-fi coexistence in unlicensed bands," in 2013 IEEE 77th Vehicular Technology Conference (VTC Spring). IEEE, 2013, pp. 1-6.

[19] V. Maglogiannis, D. Naudts, P. Willemen, and I. Moerman, "Impact of lte operating in unlicensed spectrum on wi-fi using real equipment," in Global Communications Conference (GLOBECOM), 2016 IEEE. IEEE, 2016, pp. 1-6.

[20] N. Jindal and D. Breslin, "Lte and wi-fi in unlicensed spectrum: A coexistence study," Google white paper, 2015.

[21] Qualcomm, "Qualcomm research lte in unlicensed spectrum: Harmonious coexistence with wifi," Qualcomm, Tech. Rep., 06 2014. 
[22] A. Shahid, S. Aslam, H. S. Kim, and K.-G. Lee, "Distributed 1378 joint resource and power allocation in self-organized femtocell 1379 networks: a potential game approach," Journal of Network and 1380 Computer Applications, vol. 46, pp. 280-292, 2014.

[23] W. Li, W. Zheng, T. Su, and X. Wen, "Dual-utility based green 1382 power game in two-tier ofdma femtocell networks with firefly 1383 algorithm," in Wireless Communications and Networking Conference 1384 (WCNC), 2013 IEEE. IEEE, 2013, pp. 452-457.

[24] G. Miao, N. Himayat, G. Y. Li, and S. Talwar, "Distributed 1386 interference-aware energy-efficient power optimization," IEEE 1387 Transactions on Wireless Communications, vol. 10, no. 4, pp. 1323- 1388 1333, 2011.

[25] F. Meshkati, H. V. Poor, and S. C. Schwartz, "Energy-efficient 1390 resource allocation in wireless networks," IEEE Signal Processing 1391 Magazine, vol. 24, no. 3, pp. 58-68, 2007.

[26] R. Xie, F. R. Yu, H. Ji, and Y. Li, "Energy-efficient resource 1393 allocation for heterogeneous cognitive radio networks with fem- 1394 tocells," IEEE Transactions on Wireless Communications, vol. 11, 1395 no. 11, pp. 3910-3920, 2012.

[27] A. H. Arani, A. Mehbodniya, M. J. Omidi, F. Adachi, W. Saad, 1397 and I. Güvenç, "Distributed learning for energy-efficient re- 1398 source management in self-organizing heterogeneous net- 1399 works," IEEE Transactions on Vehicular Technology, vol. 66, no. 10, 1400 pp. 9287-9303, 2017.

[28] S. Samarakoon, M. Bennis, W. Saad, M. Debbah, and M. Latva- 1402 Aho, "Ultra dense small cell networks: Turning density into en- ${ }^{1403}$ ergy efficiency," IEEE Journal on Selected Areas in Communications, 1404 vol. 34 , no. 5, pp. 1267-1280, 2016

[29] A. Zakrzewska, D. López-Pérez, S. Kucera, and H. Claussen, 1406 "Dual connectivity in lte hetnets with split control-and user- ${ }^{1407}$ plane," in Globecom Workshops (GC Wkshps), 2013 IEEE. IEEE, 1408 2013, pp. 391-396.

[30] M. R. Khawer, J. Tang, and F. Han, "usicica proactive small cell 1410 interference mitigation strategy for improving spectral efficiency ${ }^{1411}$ of lte networks in the unlicensed spectrum," IEEE Transactions on ${ }^{1412}$ Wireless Communications, vol. 15, no. 3, pp. 2303-2311, 2016.

[31] F. Liu, E. Bala, E. Erkip, M. C. Beluri, and R. "Small-cell 1414 traffic balancing over licensed and unlicensed bands," IEEE ${ }^{1415}$ Transactions on Vehicular Technology, vol. 64, no. 12, pp. 5850- ${ }^{1416}$ $5865,2015$.

[32] H. He, H. Shan, A. Huang, L. X. Cai, and T. Quek, "Proportional ${ }^{1418}$ fairness-based resource allocation for lte-u coexisting with wi- ${ }_{1419}$ fi," IEEE Access, 2016.

[33] R Etkin A Parekh and D. Tse "Spectrum sharing for unli- 142 censed bands," IEEE Journal on selected areas in communications, ${ }_{1422}$ vol. 25, no. 3, 2007.

[34] F. Teng, D. Guo, and M. L. Honig, "Sharing of unlicensed spec- ${ }^{1424}$ trum by strategic operators," in Signal and Information Processing (GlobalSIP), 2014 IEEE Global Conference on. IEEE, 2014, pp. 288- ${ }^{1426}$ 292.

[35] M. Chen, W. Saad, and C. Yin, "Echo state networks for self- ${ }^{1428}$ organizing resource allocation in lte-u with uplink-downlink ${ }_{1430}^{1429}$ decoupling," IEEE Transactions on Wireless Communications, 2016.

[36] M. Chen, W. Saad, and C. Yin, "Optimized uplink-downlink ${ }^{143}$ decoupling in lte-u networks: An echo state approach," in 2016 IEEE International Conference on Communications (ICC). IEEE, ${ }_{1434}^{1433}$ 2016, pp. 1-6.

[37] R. Thakur, V. J. Kotagi, and C. S. R. Murthy, "Resource allocation and cell selection framework for lte-unlicensed femtocell networks," Computer Networks, vol. 129, pp. 273-283, 2017.

[38] A. Shahid, K. S. Kim, E. De Poorter, and I. Moerman, "Selforganized energy-efficient cross-layer optimization for device to device communication in heterogeneous cellular networks," IEEE Access, vol. 5, pp. 1117-1128, 2017.

[39] M. Olbrich, A. Zubow, S. Zehl, and A. Wolisz, "Towards lte-u interference detection, assessment and mitigation in 802.11 networks using commodity hardware," arXiv preprint arXiv:1702.04020, 2017.

[40] P. Gawłowicz, A. Zubow, and A. Wolisz, "Enabling crosstechnology communication between lte unlicensed and wifi," in IEEE INFOCOM, 2018.

[41] V. Maglogiannis, A. Shahid, D. Naudts, E. De Poorter, and I. Moerman, "Enhancing the coexistence of lte and wi-fi in unlicensed spectrum through convolutional neural networks," IEEE Access, vol. 7, pp. 28 464-28 477, 2019.

[42] A. Shahid, J. Fontaine, M. Camelo, J. Haxhibeqiri, M. Saelens, Z. Khan, I. Moerman, and E. De Poorter, "A convolutional neu- ral network approach for classification of lpwan technologies: Sigfox, lora and ieee 802.15. 4g," in 2019 16th Annual IEEE International Conference on Sensing, Communication, and Networking (SECON). IEEE, 2019, pp. 1-8.

[43] J. Fontaine, E. Fonseca, A. Shahid, M. Kist, L. A. DaSilva, I. Moerman, and E. De Poorter, "Towards low-complexity wireless technology classification across multiple environments," Ad Hoc Networks, vol. 91, p. 101881, 2019.

[44] T. L. Marzetta, "How much training is required for multiuser mimo?" in 2006 Fortieth Asilomar Conference on Signals, Systems and Computers. IEEE, 2006, pp. 359-363.

[45] J. Wang, G. Scutari, and D. P. Palomar, "Robust mimo cognitive radio via game theory," IEEE Transactions on Signal Processing, vol. 59, no. 3, pp. 1183-1201, 2011.

[46] H. Zhang, C. Jiang, X. Mao, and H.-H. Chen, "Interferencelimited resource optimization in cognitive femtocells with fairness and imperfect spectrum sensing," IEEE Transactions on Vehicular Technology, vol. 65, no. 3, pp. 1761-1771, 2016.

[47] "Lte-u technology and coexisntance," Available at http://www.lteuforum.org/workshop.html.

[48] H. Zhang, C. Jiang, N. C. Beaulieu, X. Chu, X. Wang, and T. Q. Quek, "Resource allocation for cognitive small cell networks: A cooperative bargaining game theoretic approach," IEEE Transactions on Wireless Communications, vol. 14, no. 6, pp. 3481-3493, 2015.

[49] X. Xiao, X. Tao, and J. Lu, "Qos-aware energy-efficient radio resource scheduling in multi-user ofdma systems," IEEE Communications Letters, vol. 17, no. 1, pp. 75-78, 2013.

[50] A. Zappone, E. Jorswieck et al., "Energy efficiency in wireless networks via fractional programming theory," Foundations and Trends $(\mathbb{R}$ in Communications and Information Theory, vol. 11, no. 3-4, pp. 185-396, 2015.

[51] W. Dinkelbach, "On nonlinear fractional programming," Management science, vol. 13, no. 7, pp. 492-498, 1967.

[52] K. Akkarajitsakul, E. Hossain, D. Niyato, and D. I. Kim, "Game theoretic approaches for multiple access in wireless networks: A survey," IEEE Communications Surveys \& Tutorials, vol. 13, no. 3, pp. 372-395, 2011.

[53] J. Lee and S. Leyffer, "Mixed integer nonlinear programming. the ima volumes in mathematics and its applications, vol. 154," 2012.

[54] K. Son, S. Lee, Y. Yi, and S. Chong, "Refim: A practical interference management in heterogeneous wireless access networks," arXiv preprint arXiv:1105.0738, 2011.

[55] Z. Shen, J. G. Andrews, and B. L. Evans, "Adaptive resource allocation in multiuser ofdm systems with proportional rate constraints," IEEE transactions on wireless communications, vol. 4, no. 6, pp. 2726-2737, 2005.

[56] D. Fudenberg and J. Tirole, "Game theory," 1991.

[57] C. Patel, M. Yavuz, and S. Nanda, "Femtocells [industry perspectives]," IEEE Wireless Communications, vol. 17, no. 5, pp. 6-7, 2010.

[58] Z. Chang, Z. Han, and T. Ristaniemi, "Energy efficient optimization for wireless virtualized small cell networks with large-scale multiple antenna," IEEE Transactions on Communications, vol. 65, no. 4, pp. 1696-1707, 2017.

[59] E. U. T. R. Access, "Further advancements for e-utra physical layer aspects," 3GPP TR 36.814, Tech. Rep., 2010.

[60] I. ETS, "Selection procedures for the choice of radio transmission technologies of the umts (umts 30.03 version 3.2. 0)," Universal Mobile Telecommunications System (UMTS), 1998. 
Adnan Shahid (M15, SM17) received the 1503 B.Eng. and the M.Eng. degrees in Computer 1504 Engineering with from the University of En- 1505 gineering and Technology, Taxila, Pakistan in 1506 2006 and 2010, respectively, and the Ph.D 1507 degree in Information and Communication En- 1508 gineering from Sejong University, South Korea 1509 in 2015 . He is currently working as a Senior 1510 Researcher in IDLab, a core research group 1511 of imec with research activities combined with 1512 Ghent University and University of Antwerp. 1513 $\mathrm{He}$ is and has been involved in several ongoing and finished European 1514 research projects such as eWINE, WiSHFUL, etc and national projects 1515 such as SAMURAI, IDEAL-IOT, Cognitive Wireless Networking Man- 1516 agement etc. $\mathrm{He}$ is a senior member of IEEE and actively involved in 1517 various research activities. He is also serving as an associate editor in 1518 various journals including IEEE Access and Journal of Networks and 1519 Computer Application (JNCA). He is the author or co-author of more 1520 than 45 plus publications in well-known journals and conferences. 1521 His research interests include resource management, interference 1522 management, self-organizing networks, small cell networks, Internet 1523 of things, $5 \mathrm{G}$ wireless networks, machine learning, etc.

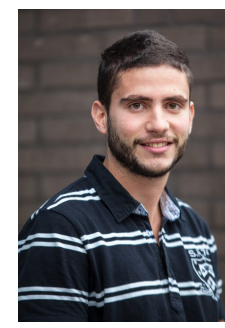

Vasilis.Maglogiannis Vasilis Maglogiannis received his B.Eng degree in Computer Engi- 1532 neering and his M.Sc in Science and Technol- 1533 ogy of Computers, Telecommunications and ${ }_{1534}$ Networks from University of Thessaly, Greece 1535 in 2012 and 2014 respectively. In 2014, he 1536 joined the IDLab of the Department of Infor- 1537 mation Technology, University of Ghent - imec, Belgium. $\mathrm{He}$ is currently pursuing his $\mathrm{PhD}$ degree in the faculty of Engineering and Architecture at University of Ghent, Belgium. His main research interests include mobile and wireless networks, 5G, LTE, $802.11,802.15 .4$, spectrum sharing and wireless sensor networks. $\mathrm{He}$ has experience in working on national, FP7 and H2020 European projects.

Irfan Ahmed (M'10, SM'16) received the B.E. Electrical Engineering degree and the M.S. Computer Engineering degree from University of Engineering and Technology, Taxila, Pakistan, in 1999 and 2003, respectively, and the $\mathrm{PhD}$ degree in Telecommunication Engineering from the Beijing University of Posts and Telecommunications, Beijing, China, in 2008. Currently, he is working as associate professor in Higher Colleges of Technology, UAE. He 1538 was post-doctoral fellow with Qatar University 1539 from April 2010 to March 2011, where he worked on two research 1540 projects, wireless mesh networks with Purdue University, USA, and 1541 radio resource allocation for LTE with Qtel. He has also been involved 1542 in National ICT Pakistan funded research project 'Design and devel- ${ }_{1543}$ opment of MIMO and Cooperative MIMO test-bed' at lqra University, 1544 Islamabad, Pakistan, during 2008 to 2010. His research interests 1545 include wireless LAN (WLAN) medium access control (MAC) protocol 1546 design and analysis, cooperative communications, MIMO communica- 1547 tions, performance analysis of wireless channels, energy constrained ${ }_{1548}$ wireless networks, cognitive radio networks, and radio resource al- 1549 location. $\mathrm{He}$ is an author of more than 25 International publications. 1550 Dr. Irfan served as session chair of the IEEE Wireless Communica- 1551 tions, Networking and Mobile Computing conference held in Shanghai, 1552 China, in September 2007, IEEE ICC 2016. He is an active reviewer 1553 of IEEE, Springer, and Elsevier journals, and conferences. He is an ${ }_{1554}$ associate editor of IEEE Access journal.

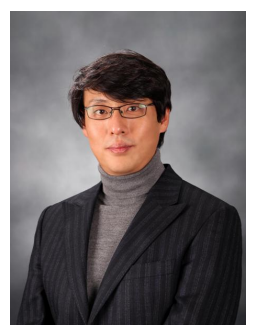

Kwang Soon Kim (S'95, M'99, SM'04) was born in Seoul, Korea, on September 20, 1972. $\mathrm{He}$ received the B.S. (summa cum laude), M.S.E., and Ph.D. degrees in Electrical Engineering from Korea Advanced Institute of Science and Technology (KAIST), Daejeon, Korea, in February 1994, February 1996, and February 1999, respectively. From March 1999 to March 2000, he was with the Department of Electrical and Computer Engineering, University of California at San Diego, La Jolla, CA U.S.A., as a Postdoctoral Researcher. From April 2000 to February 2004, he was with the Mobile Telecommunication Research Laboratory, Electronics and Telecommunication Research Institute, Daejeon, Korea as a Senior Member of Research Staff. Since March 2004, he has been with the Department of Electrical and Electronic Engineering, Yonsei University, Seoul, Korea, now is a Professor. Prof. Kim is a Senior Member of the IEEE, served as an Editor of the Journal of the Korean Institute of Communications and Information Sciences (KICS) from 2006-2012, as the Editor-in-Chief of the journal of KICS since 2013, as an Editor of the Journal of Communications and Networks (JCN) since 2008, as an Editor of the IEEE Transactions on Wireless Communications 2009-2014. He was a recipient of the Postdoctoral Fellowship from Korea Science and Engineering Foundation (KOSEF) in 1999. He received the Outstanding Researcher Award from Electronics and Telecommunication Research Institute (ETRI) in 2002 the Jack Neubauer Memorial Award (Best system paper award, IEEE Transactions on Vehicular Technology) from IEEE Vehicular Technology Society in 2008, and LG R\&D Award: Industry-Academic Cooperation Prize, LG Electronics, 2013. His research interests are generally in signal processing, communication theory, information theory, and stochastic geometry applied to wireless heterogeneous cellular networks, wireless local area networks, wireless D2D networks and wireless ad doc networks, and are focused on the new radio access technologies for $5 \mathrm{G}$, recently.

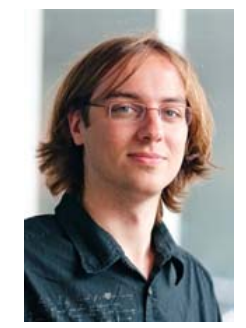

Eli De Poorter is a Professor at Ghent University. He received his master degree in Computer Science Engineering from Ghent University, Belgium, in 2006, his Ph.D. degree in 2011 at the Department of Information Technology at Ghent University and became a full-time professor at Internet and Data Lab research group (IDLab, www.IDLab.ugent.be) in 2015. In addition to teaching several courses, he is involved in and/or research coordinator of multiple national and international projects related to future wireless networks. His main research interests include wireless network protocols, network architectures, wireless sensor and ad hoc networks, future internet, self-learning networks and nextgeneration network architectures. He is part of the program committee of several conferences and is the author or co-author of more than 90 papers published in international journals or in the proceedings of international conferences. He is also the creator of the patented IDRA architecture, a flexible communication framework for heterogeneous networked devices. 


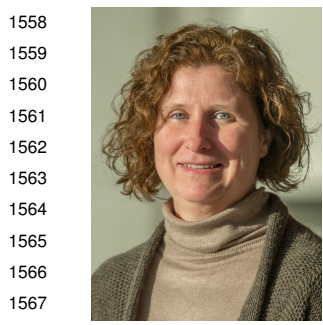

Ingrid Moerman received her degree in Electrical Engineering (1987) and the Ph.D degree (1992) from the Ghent University, where she became a part-time professor in 2000 . She is a staff member at IDLab, a core research group of imec with research activities embedded in Ghent University and University of Antwerp. Ingrid Moerman is coordinating the research activities on mobile and wireless networking, and she is leading a research team of about 30 members at IDLab-Ghent University. Her main research interests include: Internet of Things, Low Power Wide Area Networks (LPWAN), High-density wireless access networks, collaborative and cooperative networks, intelligent cognitive radio networks, real-time software defined radio, flexible hardware/software architectures for radio/network control and management, and experimentallysupported research. Ingrid Moerman has a longstanding experience in running and coordinating national and EU research funded projects. At the European level, Ingrid Moerman is in particular very active in the Future Networks research area, where she has coordinated and is coordinating several FP7/H2020 projects (CREW, WiSHFUL, eWINE, ORCA) and participating in other projects (Fed4FIRE, FORGE, FLEX, Flex5Gware). Ingrid Moerman is author or co-author of more than 700 publications in international journals or conference proceedings. 